\title{
Two Distinct Tumorigenic Processes in Endometrial Endometrioid Adenocarcinoma
}

Yuko Sugiyama, ${ }^{* \dagger}$ Osamu Gotoh, ${ }^{\dagger}$ Nobuyuki Fukui, ${ }^{\dagger}$ Norio Tanaka, ${ }^{\dagger}$ Katsuhiko Hasumi, ${ }^{*}$ Yutaka Takazawa, ${ }^{\dagger}$ Tetsuo Noda, ${ }^{\dagger}$ and Seiichi Mori

From the Divisions of Gynecology* and Pathology, ${ }^{\ddagger}$ Cancer Institute Hospital, and the Cancer Precision Medicine Center, ${ }^{\dagger}$ Japanese Foundation for Cancer Research, Tokyo, Japan

Accepted for publication September 12, 2019.

Address correspondence to Seiichi Mori, Ph.D., M.D., Cancer Precision Medicine Center, Japanese Foundation for Cancer Research, 3-8-31 Ariake, Koto-ku, Tokyo, Japan. E-mail: seiichi.mori@jfcr. or.jp.

\begin{abstract}
Endometrial endometrioid adenocarcinoma (EEA) is conventionally considered to be a single pathologic entity that develops through a hyperplasia-carcinoma sequence under the influence of estrogen. Previously, another EEA subtype was described and proposed to arise directly from normal endometrium. These conventional and de novo subtypes are designated groups 1 and 2, respectively. To identify the molecular mechanisms of these distinct tumorigenic processes, we conducted comprehensive integrated analyses of genomic data with hormonal status for group 1 paired carcinoma and hyperplasia and group 2 carcinoma samples. Although group 1 carcinomas mostly exhibited genomically stable characteristics and the activation of estrogen signaling, group 2 EEAs showed enriched hypermutator and $\mathrm{CpG}$ island methylator phenotypes. Pairwise comparisons of hyperplasia and carcinoma, along with time-course analyses of the hyperplasia-carcinoma sequence, revealed the acquisition of driver mutations in the evolutionary process of hyperplasia but not in the transition from hyperplasia to carcinoma. The current study confirms the existence of two different histopathologic programs during EEA development that harbor distinct molecular bases and demonstrates the biological relevance of these differential tumorigenic processes. (Am J Pathol 2020, 190: 234-251; https://doi.org/10.1016/j.ajpath.2019.09.022)
\end{abstract}

Endometrial cancer is the most common gynecologic malignancy in industrialized countries, with the incidence increasing globally. In the widely accepted dualistic model, endometrial cancer is divided into two clinical/epidemiological entities: type 1 cancers, which occur in young and obese patients, are associated with excess estrogen, a favorable prognosis, and endometrioid histology, and are often accompanied by and/or following endometrial hyperplasia (EH); versus type 2 cancers, which represent tumors that arise in older and nonobese patients, are related to poor prognosis and nonendometrioid histotypes, and are typically of serous histology, without associated hyperplastic lesions. $^{1-6}$ Endometrial endometrioid adenocarcinoma (EEA) has been conventionally thought to develop from $\mathrm{EH}$ as a precursor lesion through a process called hyperplasiacarcinoma sequence. Although signs of hyperestrogenism-as exemplified by the presence of $\mathrm{EH}$ and delayed menopause-are typical features of patients with EEA, ${ }^{3-6}$ a handful of previous studies have described a nonnegligible fraction of patients with EEAs (17\% to $77 \%$ ) who microscopically lack concurrent hyperplasia ${ }^{7-12}$; such EEAs are proposed to arise de novo from background normal, but often atrophic, endometria of menopaused women. In line with previous literature, ${ }^{2,12}$ EEAs with and without $\mathrm{EH}$ are herein designated group 1 and 2 EEAs, respectively. Although patients with group 2 EEA are less likely to have hyperestrogenic phenotypes - similar to that seen with type 2 endometrial cancer ${ }^{8,10-12}$ - the biological and clinicopathologic properties and tumorigenic processes of group 2 EEAs are largely unknown. Similarly, despite previous endeavors to understand the molecular

Supported by Japan Society for the Promotion of Science, Grants-in-Aid for Scientific Research (JSPS KAKENHI) grants JP17K18337 (O.G), JP15K06861 (S.M.), JP18K07338 (S.M.), JP26462543 (Y.S.), and JP17K11308 (Y.S.); and Vehicle Racing Commemorative Foundation grants 5144 (S.M.), 5274 (S.M.), and 5393 (S.M.).

Y.S. and O.G. contributed equally to this work.

Disclosures: None declared. 
mechanisms involved in the development of conventional group $1 \mathrm{EEA},{ }^{3-5,13}$ the processes involved in the transitions from normal endometrium to $\mathrm{EH}$ and from $\mathrm{EH}$ to EEA remain unclear.

Numerous previous studies have identified recurrent somatic mutations in well-described cancer genes, including PTEN, PIK3CA, ARIDIA, CTNNB1, and KRAS, which can drive EEA tumorigenesis. ${ }^{5}$ Several reports using nextgeneration sequencing technology have confirmed the significance of these driver alterations in EEA. ${ }^{13-18}$ Others have also highlighted that EH shares several molecular aberrations with EEA. ${ }^{19-25}$ Such aberrations in EH include truncating mutations in PTEN with high frequency (21\% to 55\%) and activating mutations in PIK3CA and KRAS with lower frequencies $(0 \%$ to $7 \%$ and $10 \%$ to $22 \%$, respectively $)^{19-25}$; these findings were recently confirmed in two next-generation sequencing studies. ${ }^{14,17}$ These observations suggest the likelihood of stepwise alterations to driver genes in the hyperplasia-carcinoma sequence. ${ }^{26}$ Nevertheless, genomic alterations along this sequence remain to be elucidated, presumably because few pairwise analyses have been performed on hyperplasia and carcinoma from the same patient. ${ }^{25,27}$ In addition, no sequential time-course study is available thus far. Such genomic information would help to gain an understanding of the biological underpinnings of group 1 EEA tumorigenesis and to determine possible treatment strategies for $\mathrm{EH}$.

Profiling the pattern of somatic genomic aberrations by The Cancer Genome Atlas (TCGA) identified four molecular subtypes in endometrial cancer: polymerase $\varepsilon$-mutated (POLE), microsatellite instability (MSI), copy number high $(\mathrm{CNH})$, and copy number low $(\mathrm{CNL})$ subtypes. ${ }^{13}$ These molecular subtypes were shown to be tightly linked with histotype (endometrioid or serous), histologic grade ( $1 / 2$ or 3 ), patient prognosis, and other clinicopathologic characteristics. ${ }^{13,28,29}$ More important, tumors with endometrioid histology with the same histologic grade were still heterogeneous and could be subdivided into these four subgroups. ${ }^{13,28,29}$ Because genomic aberrations are derived from various forms of DNA repair or proofreading deficiencies, each tumor belonging to the POLE, MSI, or CNH subtype-exhibiting a hypermutator phenotype-often has a causative defect in a corresponding DNA repair/proofreading system. POLE tumors are characterized by a substantial number of single-nucleotide variants (SNVs) due to mutations in the exonuclease domain of the gene coding for DNA polymerase $\varepsilon$ (POLE), which leads to defective proofreading in DNA synthesis. Tumors with the MSI subtype exhibit a high proportion of insertions/deletions (indels) caused by MLH1 gene silencing, with hypermethylation of the promoter or germline/somatic inactivation of DNA mismatch repair genes, such as $M S H 2, M S H 6$, and PMS2. Elevated copy number $(\mathrm{CN})$ abnormality is a predominant characteristic of the $\mathrm{CNH}$ subtype, which coincides frequently with mutated TP53 and less frequently with germline/ somatic mutations and epigenetic changes in the genes of the homologous recombination repair pathway components, including BRCA1 and BRCA2. ${ }^{13,30,31}$ Endometrial $\mathrm{CNH}$ tumors are typically serous but occasionally exhibit endometrioid histology. ${ }^{32,33}$ EEAs with CNL are genomically stable without any DNA repair deficiency but transcriptomically exhibit estrogen activation. ${ }^{13}$ Because DNA repair/proofreading deficiency is a relevant contributor to the tumorigenic process, ${ }^{34}$ it would be informative to be able to relate this molecular subtyping scheme with such histologic tumorigenic subgroups.

The aims of this study are twofold: first, to identify the clinical and biological differences between group 1 and 2 tumorigenic processes; and, second, to detail the stepwise emergence of driver events in the hyperplasia-carcinoma sequence during group 1 EEA development. Herein, we address these questions by analyzing genomic and epigenomic data with hormonal status derived from synchronous pairs of group 1 hyperplasia and carcinoma, together with time-course sequential sampling, and group 2 carcinoma samples. The current study highlights a differential mutational burden for the tumorigenic programs associated with groups 1 and 2 and the acquisition of driver mutations in the evolutionary process of hyperplasia but not in the transition from EH to EEA.

\section{Materials and Methods}

\section{Ethical Approval}

Ethical approval was obtained from internal review boards of the Japanese Foundation for Cancer Research. Recruited patients provided written informed consent.

\section{Histopathologic Diagnosis}

Pathologic diagnosis and classification of endometrial carcinoma were performed on the basis of the World Health Organization Classification of Tumors $2003^{35}$ by three independent gynecologic pathologists (Y.S., K.H., and Y.T.). Staging was performed according to the 2008 modified International Federation of Gynecology and Obstetrics system. ${ }^{36}$ Pathologic definition of endometrial hyperplasia (EH) was as previously described. ${ }^{37}$ Differential diagnosis for hyperplasia and carcinoma was performed according to the criteria of Silverberg and Kurman. ${ }^{38}$ Tumorigenic subtypes of endometrioid carcinoma were rigorously evaluated by the presence or absence of EH adjacent to the carcinoma in the entire endometrium of the uterus after hysterectomy, as previously described. ${ }^{12}$ Because carcinoma can grow into and over adjacent hyperplasia, smaller EEAs $(<15 \mathrm{~mm}$ along the major axis) were selected to minimize misclassifications. Although each of the 35 group 1 tissues contained EH without atypia, 22 also included atypia lesions. Atypical hyperplasia was removed with laser-capture microdissection, and all genomic assays for hyperplasia samples were performed only using EH without atypia. Patient clinical 
characteristics were obtained through medical records. Patients had not received chemotherapy or radiation therapy before surgical treatment. Four patients (three in group 1; one in group 2) were treated with oral progestin.

\section{Endometrial Carcinoma Samples and Sample \\ Preparation for Transcriptomic, Genomic, and Epigenetic Analysis}

Surgical EEA specimens were dissected and processed for histopathologic or immunohistochemical examinations (formalin fixed) as well as exome, methylome, and transcriptome analyses (snap frozen) (Supplemental Tables S1 and S2). Endometrial samples for the time-course exome study were obtained from endometrial curettage and were formalin fixed and paraffin embedded (FFPE).

Frozen tumor tissues were cut into sections (10 $\mu \mathrm{m}$ thick). Laser-capture microdissection with an LMD7000 microscope (Leica, Wetzlar, Germany) was used to dissect carcinoma from hyperplasia in group 1 tumors, enrich for cancer cells in group 2 tumors, and distinguish EH without atypia from $\mathrm{EH}$ with atypia, as described above.

DNA was extracted from tumor/matched normal tissue samples and whole blood using a QIAamp DNA Micro Kit (Qiagen, Hilden, Germany) and checked using the NanoDrop 2000 (ThermoFisher, Waltham, MA) and Qubit 2.0 fluorometer (ThermoFisher). DNA samples of appropriate purity $\left(\mathrm{OD}_{260 / 280 \mathrm{~nm}}>1.7\right)$ and concentration (ratio of doublestranded DNA/single-stranded DNA concentration > 0.35) were further processed for exome and DNA methylation microarray analyses. RNA from carcinoma and hyperplasia lesions was extracted using the RNeasy Kit (Qiagen) and checked using the NanoDrop 2000 and the 2100 Bioanalyzer (Agilent, Santa Clara, CA). Selected RNA samples (RNA purity: $\mathrm{OD}_{260 / 280 ~ n m}>1.7$; and integrity $>5.0$ ) were further processed for RNA expression microarray analyses.

\section{Immunohistochemical Evaluation of Estrogen and Progesterone Receptors}

For immunohistochemistry, sections $(4 \mu \mathrm{m}$ thick) from FFPE tissue samples were stained using an automated slide staining system and detection kit (Ventana Medical Systems, Inc., Tokyo, Japan). Anti-estrogen receptor (ER) rabbit monoclonal antibody [CONFIRM anti-ER (SP1); Ventana Medical Systems, Inc.] and anti-progesterone receptor (PR) rabbit monoclonal antibody [CONFIRM antiPR (1E2); Ventana Medical Systems, Inc.] were used. An immunoreactivity score was used for positive staining: positive nuclei score ( 0 indicates no staining; $1,1 \%$ to $10 \%$ of tumor nuclei; $2,11 \%$ to $50 \%$ of tumor nuclei; $3,51 \%$ to $80 \%$ of tumor nuclei; and $4, \geq 81 \%$ of tumor nuclei) multiplied by the staining intensity score $(0$ indicates negative; 1 , weakly positive; 2 , moderately positive; and 3 , strongly positive). ${ }^{39}$
Library Preparation and Sequencing for Exome Analysis

A total of 187 specimens (34 group 1 normal/hyperplasia/carcinoma trios plus 1 group 1 normal/carcinoma pair and 34 group 2 normal/carcinoma pairs) (Supplemental Tables S1 and S2) and 15 time-course samples of hyperplasia and carcinoma from five group 1 cases were subjected to exome sequencing analyses using the SureSelect Human All Exon V4 or V5 (Agilent Technologies, Santa Clara, CA) system. The KAPA HyperPlus Kit (KAPA Biosystems, Wilmington, MA) was used to construct libraries from DNA from FFPE tissues. Captured DNA was multiplexed and sequenced with a HiSeq2500 or HiSeq2000 (Illumina, San Diego, CA), with a median coverage of 222 to 363 reads per tumor, 112 to 191 reads per normal sample, and 132 to 300 reads per FFPE sample.

\section{Bioinformatical Tools to Analyze Sequencing Data}

Sequenced reads were aligned to the reference human genome (hg19) with Burrows-Wheeler Aligner version 0.6.1. ${ }^{40}$ GenomeAnalysisTK (GATK) version $1.5-30{ }^{41}$ was used to recalibrate the variant quality score and to perform local realignment.

Somatic SNVs were called with VarScan version 2.3.7, ${ }^{42}$ MuTect version 1.1.4, ${ }^{43}$ and Karkinos version 3.0.22 (http:// sourceforge.net/projects/karkinos, last accessed August 27, 2019). ${ }^{44}$ VarScan version 2.3.7, SomaticIndelDetector version 1.5-30, ${ }^{45}$ and Karkinos version 3.0.22 were used to detect somatic indels. Somatic SNVs and indels were taken as genuine mutations when found at least twice among the three callers. Somatic copy number variants were detected by EXCAVATOR version 2.2. ${ }^{46}$ GISTIC version 2.0.22 was used to present altered copy numbers as a heat map, along with chromosome position. Aberrant $\mathrm{CN}$ changes were defined as gain $(\mathrm{CN} \geq 3)$, amplification $(\mathrm{CN} \geq 4)$, loss $(\mathrm{CN}=1)$, and homozygous deletion $(\mathrm{CN}=0)$.

ExomeCNV version $1.4^{48}$ was used to infer tumor content for group 1 hyperplasia and carcinoma and group 2 carcinoma. There was no statistical difference in median tumor content (0.782, 0.761, and 0.726 , respectively).

Tumor mutational burden per megabase in the captured exome was computed as described. ${ }^{49}$ The number of missense SNVs per sample was counted and divided by the number of bp $(30,435,778 \mathrm{bp})$ in the captured regions of the coding exome.

\section{Molecular Classification of EEA Samples by TCGA Subtyping Scheme}

EEA classification was performed using the molecular subtyping scheme developed by TCGA. ${ }^{13}$ Samples assigned to the POLE subtype had mutations in the exonuclease domain (residues 286 to 459 in the amino acid sequencing) of the POLE protein. Microsatellite instability high (MSI subtype) tumors were assigned on the basis of deviations from paired normal control in electropherograms of two or more among six 
DNA markers (BAT25, BAT26, D2S123, D5S346, D17S250, and BAT40). ${ }^{50} \mathrm{CNH}$ subtype tumors were annotated on the basis of their similarity to the cluster $4-$ like cluster ${ }^{13}$ in the unsupervised hierarchical clustering analysis, with significantly altered copy number segment values identified with GISTIC. $^{51}$ The remaining EEA samples, after excluding POLE, MSI, and CNH, were designated as CNL subtype. ${ }^{13}$

\section{Driver and Passenger Genes and Mutations}

Driver genes were defined for endometrial cancer as highly significantly mutated or significantly mutated genes, according to the TumorPortal (http://www.tumorportal.org, last accessed August 27, 2019). ${ }^{52}$ Because a mutation on a significantly mutated gene is not necessarily a driver mutation, it was assumed that detected variants were more likely to be driver mutations if they were at least registered once previously in the TumorPortal or Catalogue Of Somatic Mutations In Cancer (COSMIC) database.

\section{Filters for FFPE Samples in Time-Course Analysis}

Sequencing noise (ie, caused by damaged DNA in FFPE samples) was filtered by removing the following: i) mutant alleles called at poorly mapped reads (mapping quality $<30$ ); ii) mutant alleles with a read depth $<50$; iii) indels called at the edge of homopolymeric nucleotides (more than four of the same successive nucleotides); and iv) recurrent mutant alleles across cases in the cohort but not recurrent in the TumorPortal or COSMIC database. Mutant alleles on aneuploid chromosomes were also removed to evaluate allele frequency. The same filters were applied to sequencing data from fresh frozen samples. Hyperplasia or carcinoma sample reads from each patient in the time-course series were subjected to pairwise local realignment using GATK along with a matched normal sample to reduce erroneous calls by misalignment. ${ }^{45}$

\section{Time-Course Analysis of Clonal Architecture}

Herein, a subclone was defined in a tumor by its allele frequencies as a cluster of reads with somatic mutations. After filtering (described above), mutant allele frequencies for the remaining somatic SNVs/indels in each of the time-course samples from each patient were first clustered using unsupervised hierarchical clustering [with euclidean distance and Ward linkage; R software version 3.5.1 (http://www.r-project.org, last accessed August 27, 2019)]. Because unsupervised hierarchical clustering has poor resolution in clustering variants with low allele frequencies, SciClone version 1.0.7 (minimal depth $=50$, maximal number of clusters $=10$, and copy number margins $=0.25)^{53}$ was also used when maximal mutant allele frequency in a cluster was $<0.2$. The resultant clusters were then manually compiled. After this, 7 to 10 (median, 8) clusters in a tumor series were assigned in one case. The nested relationships among clusters were estimated on the basis of cluster size and the spatiotemporal fluctuations of each cluster. Adobe Illustrator and Photoshop (CS6) (both from Adobe, San Jose, CA) were used to visualize the relationship among clones/subclones in each case, as previously described. ${ }^{54}$

\section{DNA Methylome Analysis}

DNA was prepared from 34 pairs of group 1 hyperplasia and carcinoma (plus one carcinoma missing the adjacent hyperplasia data) and 34 group 2 carcinomas (Supplemental Tables S1 and S2). DNA methylation status was analyzed using 500 ng DNA from each of the 103 tumors using Infinium MethylationEPIC BeadChip Arrays (Illumina), according to the manufacturer's instructions. All arrays fulfilled the experimental criteria for each experimental step (staining, extension, hybridization, target removal, and bisulfite conversion). Fluorescence signals were converted into $\beta$ values using Illumina Genome Studio software version 2011.1 after background subtraction and normalization. Missing values were filtered from a total of 866,895 probes, with signals from 750,190 probes used for subsequent analyses.

$\mathrm{CpG}$ methylation in the promoter region of $M L H 1$ was determined by first selecting inversely correlated probes with $M L H 1$ expression with correlation coefficients $<-0.8$ (Pearson correlation). Variably methylated probes were then selected with the variance of $\beta$ values $>0.025$, and $M L H 1$ promoter hypermethylation was determined if the mean $\beta$ value was $>0.5$. Highly variably methylated probes (top 6963 probes showing top 5\% variance on $\mathrm{CpG}$ islands of promoter regions) were subjected to unsupervised consensus clustering using R with Bioconductor ConsensusClusterPlus.

DNA methylation target genes were selected if they met the following criteria: i) genes with probes on $\mathrm{CpG}$ islands and/or with annotations of differentially methylated regions; ii) genes with variably methylated probes $(\beta$ value median $<0.1)$; iii) genes with probes inversely correlated with the expression value of the gene (Spearman correlation $\rho<-0.65$ ); iv) genes with probes for which more than a half of the samples were hypomethylated (median $\beta$ value $<0.1$ ); and v) genes with more than five probes that also sufficed the conditions of i) through iv). The degree of gene expression silencing due to DNA methylation (\% silencing) was calculated as follows: $(100 \%$ - the $\%$ of the given expression value of each gene for a sample)/(the maximal expression value across the samples).

\section{Expression Microarrays}

Expression assays were performed with Affymetrix Human U133 Plus 2.0 arrays (Affymetrix, Santa Clara, CA) using RNA extracted from 33 group 1 hyperplasias, 31 group 1 carcinomas (30 pairs of concomitant hyperplasia and carcinoma from the same patients with an additional 3 unpaired hyperplasia and 1 unpaired carcinoma) and 33 group 2 carcinomas (Supplemental Tables S1 and S2). All arrays met the standard quality control metrics, including hybridization 
controls, labeling controls, global array metrics, and algorithm parameters, and were used to compute robust multichip average expression values using Affymetrix Expression Console software version 1.1.2. All expression values on the arrays were used in further analyses without filtering.

To identify group 1 or 2 specific pathway enrichment in the transcriptome, single-sample Gene Set Enrichment Analysis (ssGSEA) was performed on expression microarray data using $\mathrm{R}$ software with Bioconductor gene set variation analysis for microarray and RNA-seq data (GSVA) and the Molecular Signature DataBase version 5.0 55 (http://software. broadinstitute.org/gsea/msigdb/index.jsp, last accessed August 27, 2019; Broad Institute, Cambridge, MA). The data set was first collapsed into gene symbols, and genes were ranked by the signal/noise ratio metric for phenotypes with 1000 permutations. The hypergeometric distribution test was employed to determine whether an annotation of interest (such as DNA methylation) was enriched in a group. As a threshold for the distribution, $q<0.05$ computed from significance analysis of microarrays (SAM) was used in a binary comparison.

For transcriptomic subtyping, consensus clustering ${ }^{58}$ was employed to identify clusters corresponding to internal subgroups in EEA using $\mathrm{R}$ with Bioconductor ConsensusClusterPlus. ${ }^{59}$ On the basis of variance in the expression across samples, 4889 genes (top 19\% variable expression, selected by pvclust in R package) were selected and used for k-means clustering with euclidean distance and a subsampling ratio of 0.8 for 1000 iterations. Ingenuity Pathway Analysis version $458397 \mathrm{M}$ was employed to annotate a cluster of genes with the top canonical pathways.

\section{Statistical Analysis}

$U$-test and Fisher exact test were used to statistically evaluate the correlation between clinicopathologic parameters and EEA groups using GraphPad Prism version 8.3.0 (GraphPad Software, San Diego, CA) or R software.

\section{Data Access}

The raw data generated in this study have been submitted to the National Bioscience Database Center [https://ddbj.nig. ac.jp/jga/viewer/view/study; accession number JGAS00000000174 (Exome BAM files)] and National Center for Biotechnology Information Gene Expression Omnibus [https://www.ncbi.nlm.nih.gov/geo; accession numbers GSE106191 (RNA expression microarray cell intensity files) and GSE136791 (DNA methylome microarray intensity data files)].

\section{Results}

\section{A Classification Scheme of Endometrial Endometrioid Adenocarcinoma and Samples}

Among the 1616 endometrial carcinomas that were surgically removed at the Cancer Institute Hospital (Tokyo,
Japan) between 1986 and 2013, 1381 cases were diagnosed as EEA. After size $(<15 \mathrm{~mm})$ selection by microscopic examination, 212 cases were determined eligible for the current study. The tumor specimens were subsequently assessed for the presence or absence of endometrial complex hyperplasia (regardless of atypia) in the endometria adjacent to EEA. ${ }^{12}$ Of the 212 cases, 104 and 108 cases were classified as group 1 (with $\mathrm{EH}$ ) and group 2 (without $\mathrm{EH}$ ), respectively. Among the clinical characteristics for these 212 patients, statistically significant differences between two groups were observed for age at diagnosis, body mass index, menopausal status, and histologic grade: patients with group 1 EEA were younger at age of onset, had higher body mass indexes, and were more often premenopausal, whereas group 2 EEAs comprised a higher number of highgrade carcinomas (Figure 1). These distinctions are reminiscent of the results from previous histopathologic classification studies. ${ }^{8,11,60}$ There were no differences in other parameters, such as number of gravida and partus, or International Federation of Gynecology and Obstetrics staging (Figure 1) (data not shown). Likewise, there was no difference in the proportions of metabolic complications, such as hypertension or diabetes mellitus, which are typical risk factors for EEA (data not shown). ${ }^{12}$ Patient outcomes in terms of 5-year survival were equally good for both groups, probably because most patients were diagnosed at clinical stage 1 (data not shown). After selecting tumors on the basis of histologic grade (1 or 2), fresh-frozen tumor samples were processed for further genomic analyses (Figure 1). Although there was no significant difference in the distribution of International Federation of Gynecology and Obstetrics stage between the two groups, group 2 included more higher-grade carcinomas among the samples in the whole cohort and in those samples subjected to genomic analysis (Figure 1). To gain deeper insight into the developmental processes of these two distinct EEA tumorigenic subtypes, genomic, epigenetic, and transcriptomic analyses were conducted with stringent histopathologic classification (Figure 1). In total, 35 carcinoma and 34 hyperplasia samples from 35 cases of group 1 EEAs (each case was paired, except for one case with insufficient hyperplasia sample for genomic analyses) and 34 carcinomas from 34 cases of group 2 EEAs were subjected to genomic assays (Figure 1 and Supplemental Tables S1 and S2). There were 29 and 6 histologic grade 1 and 2 carcinomas, respectively, in group 1 samples and 21 and 13 grade 1 and 2 tumors, respectively, in group 2 samples $(P=0.0499$ by Fisher exact test) (Figure 1). All histologic grade 3 carcinomas included in group 2 were not subjected to genomic analyses.

\section{Genomic Aberration Profiles in Group 1 and 2 Carcinomas}

In exome analyses, highly variable genomic aberration profiles across the EEA samples in terms of the number of SNVs, indels, and abnormal copy number segments 


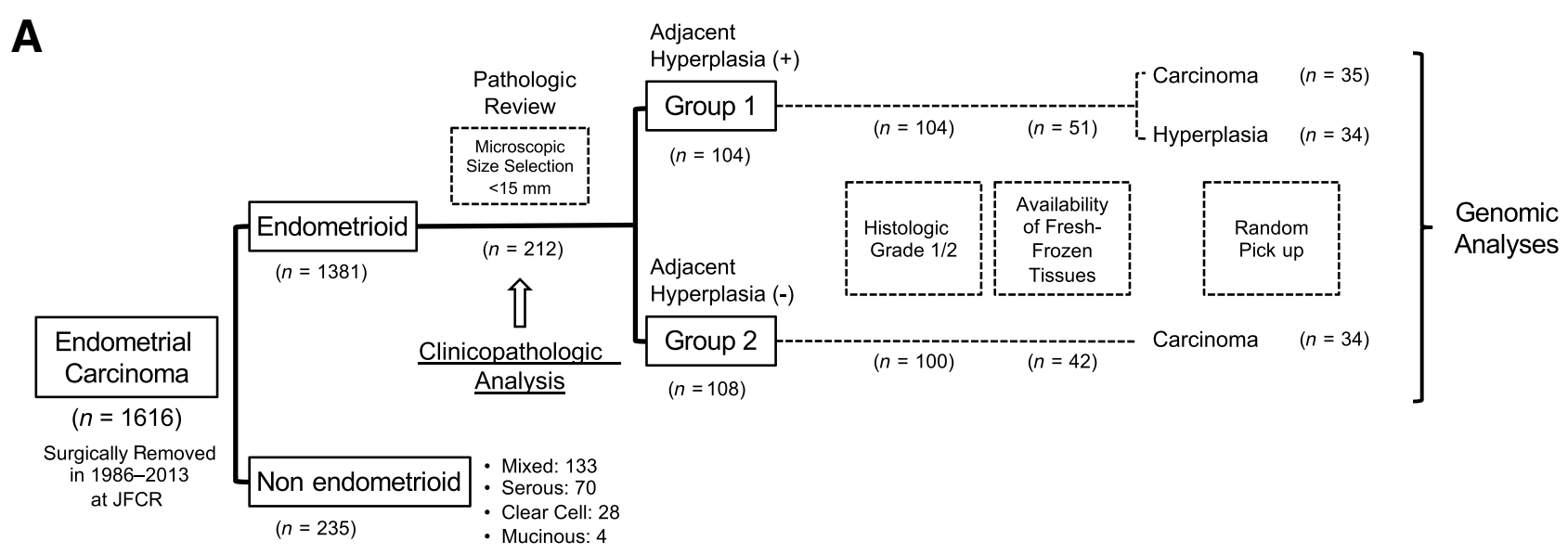

B

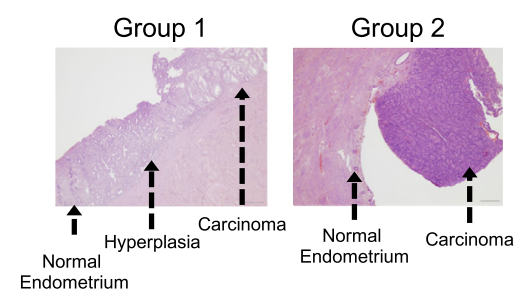

C
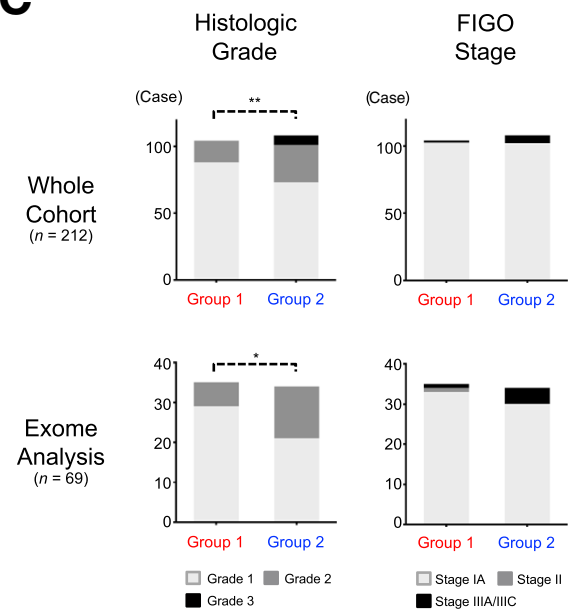

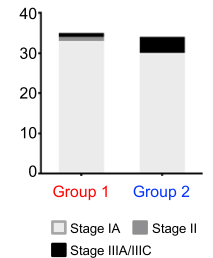

Figure 1 Genomic analyses of endometrial endometrioid adenocarcinoma (EEA) that develops through two distinct tumorigenic pathways. A: Classification scheme of endometrial carcinoma and the current genomic study design. The scheme presents a method for classification based on histopathologic properties of the tumor. Among 1381 type 1 EEAs, 212 cases passed the eligibility criteria, for which tumor size must be $<15$ mm in diameter on microscopic examination. These cases were subjected to clinicopathologic analysis. On the basis of the presence or absence of adjacent hyperplasia, 104 and 108 EEAs were then classified as group 1 and 2 tumors, respectively. A total of 34, 35, and 34 exome, 33, 31, and 33 transcriptome (with expression microarrays), and 34, 35, and 34 DNA methylome analyses were conducted on group 1 paired hyperplasia and carcinomas and group 2 carcinomas, respectively. All three genomic assays were performed for 31 group 1 and 33 group 2 cases (Supplemental Tables S1 and S2). B: Representative histopathology of group 1 (left panel) and group 2 (right panel) EEAs. Sections were stained with hematoxylin and eosin. C: Histologic grade and International Federation of Gynecology and Obstetrics (FIG0) stage. Top row: Cases for the whole cohort with clinicopathologic analysis are shown. Bottom row: Cases with genomic analysis are shown. $P$ values were calculated with Fisher exact test. $n=212$ in total (104 group 1 and 108 group 2 patients; C, top panels); $n=69$ in total (35 group 1 and 34 group 2 patients; C, bottom panels). ${ }^{*} P<0.05,{ }^{*} P<0.01$. Scale bar $=500 \mu \mathrm{m}$ (B).

(Figure 2A), and significant differences between group 1 and 2 carcinomas (Figure 2B), were detected: group 2 carcinomas had a higher number of SNVs and indels than group 1 carcinomas $(P<0.0001$ and $P=0.0061$, respectively) (Figure 2B), but similar proportions of abnormal copy number segments $(P=0.5319)$ (Figure 2B). Accordingly, tumor mutational burden per megabase in the captured exome ${ }^{49}$ was higher for group 2 carcinomas (Figure 2C). This distinction prompted us to apply TCGA subtyping to classify EEA samples in the current cohort (herein designated as the Japanese Foundation for Cancer Research genomic cohort). Of the 69 carcinomas in the Japanese Foundation for Cancer Research cohort (35 and 34 group 1 and 2 carcinomas, respectively) 11, 14, 3, and 41 carcinomas were assigned to the POLE, MSI, CNH, and
CNL subtypes, respectively (Figure 2D). These subtypes are consistent with genetic and/or epigenetic abnormalities found in genes for mismatch repair or homologous recombination pathways (data not shown). When TCGA samples were used at stage 1 and grade $1 / 2$ for comparison, the proportions of the four molecular subtypes in the Japanese Foundation for Cancer Research cohort did not differ from those in TCGA cohort (Figure 2D). ${ }^{13}$ However, the proportions were significantly different between group 1 and 2 carcinomas: Most $(31 / 35 ; 88.6 \%)$ of the group 1 carcinomas were classified as CNL subtype, whereas many (24/34, $70.6 \%$; Fisher, $P<0.0001)$ of the group 2 carcinomas were classified as subtypes other than CNL (POLE, MSI, and $\mathrm{CNH}$ ) (Figure 2D). These observations imply a differential mutational burden between group 1 and group 2 carcinomas 
A
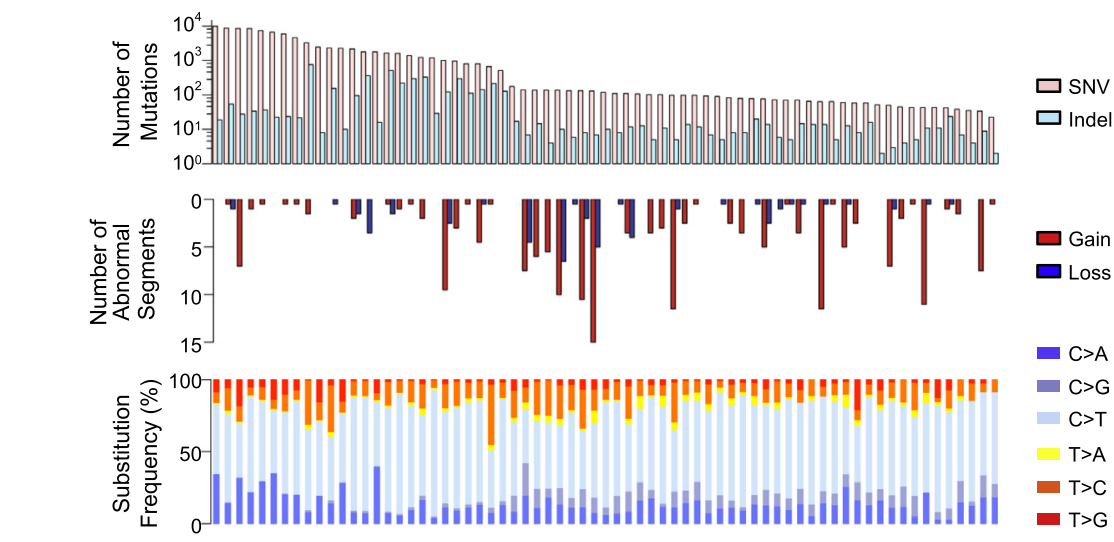

B
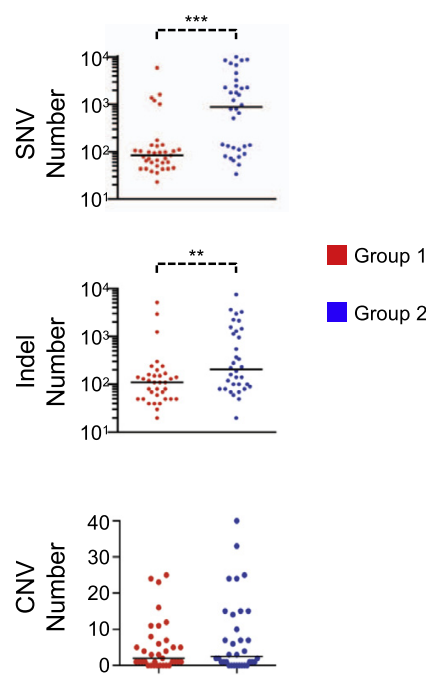

C

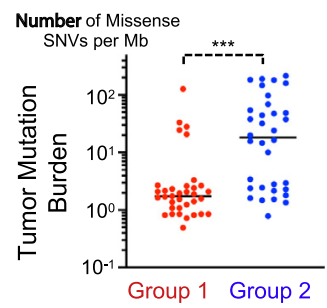

D

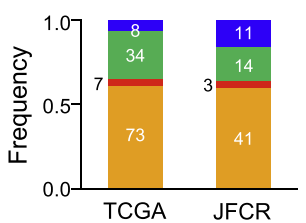

Figure 2 Genomic aberration profiles of group 1 and group 2 endometrial endometrioid adenocarcinomas (EEAs). A: Relationship of genomic deregulation in group 1 and 2 carcinomas. Number of single-nucleotide variants (SNVs) and insertions/deletions (indels; top panel), number of abnormal copy number (CN) segments ( $C N \leq 1$ or $\geq 3$; middle panel), and frequency of nucleotide substitutions (bottom panel), sorted according to the number of SNVs, are shown with sample labeling for tumorigenic and The Cancer Genome Atlas (TCGA) subtypes (bottom). B: Differential mutational burden in group 1 and 2 tumors. Top panel: Number of SNVs. Middle panel: Number of indels. Bottom panel: Number of copy number variants (CNVs). CNV number is the number of abnormal CN segments that were counted if $\mathrm{CN} \leq 1$ or $\geq 3$. The discontinuous distribution of the numbers of SNVs, indels, and CNVs is associated with TCGA molecular subtype. Statistical binary comparison was performed with $U$-test. C: Tumor mutational burden in group 1 and 2 tumors. The tumor mutational burden in the captured exome (number of SNVs per megabase) per sample is shown as dot plots. Statistical binary comparison was performed with U-test. D: Distribution of TCGA molecular subtypes [polymerase $\varepsilon$ mutated (POLE), microsatellite instability (MSI), ${ }^{13}$ copy number high (CNH), and copy number low (CNL)] in group 1 and 2 carcinomas. Left panel: TCGA subtype components in 122 TCGA (only grade 1 and 2 tumors at clinical stage 1) and 69 Japanese Foundation for Cancer Research (JFCR) EEA samples. Right panel: TCGA subtype components in 35 group 1 and 34 group 2 carcinoma samples are shown as a stacked bar chart. POLE, MSI, and CNH EEAs are predominantly enriched in group 2 tumors, whereas most of the carcinomas in group 1 exhibit CNL characteristics $(P<0.0001$; Fisher exact test). ${ }^{* *} P<0.01,{ }^{* * *} P<0.001$.

and suggest the potential involvement of DNA repair/ proofreading deficiencies in these distinct tumorigenic processes.

\section{Distribution of Driver Mutations in Group 1 and 2 Carcinomas}

It was next determined whether a specific gene mutation(s) drives the differential EEA tumorigenic programs of group 1 and 2 carcinomas. Binary comparisons using Fisher exact tests identified a significant number of mutated genes in SNVs and indels, which were correlated with group 2 carcinomas $(P<0.05 ; 1875$ genes among total 16,102 genes) but not with group 1 carcinomas. Because these group 2-correlated gene mutations were dominantly derived from hypermutator tumors of the
POLE or MSI subtypes (Figure 2D), the somatic variants enriched in group 2 (Figure 3) are likely to be passengers. In support of this notion, no significant difference was detected between group 1 and 2 CNL samples ( $n=31$ and $n=10$, respectively; data not shown). This group 2 dominancy was lost for more significant driver genes (significantly mutated and highly significantly mutated genes in TumorPortal), particularly for the four endometrioid genes (PTEN, PIK3CA, CTNNB1, and $K R A S)^{61}$ (Figure 3). In other words, there was a less-biased distribution of more relevant driver genes among group 1 and 2 tumors. No significant difference was found in copy number aberrations between the two groups (Figure 3). Collectively, these observations imply that group 1 and 2 EEAs rely on the same or similar sets of driver events during tumorigenesis. 


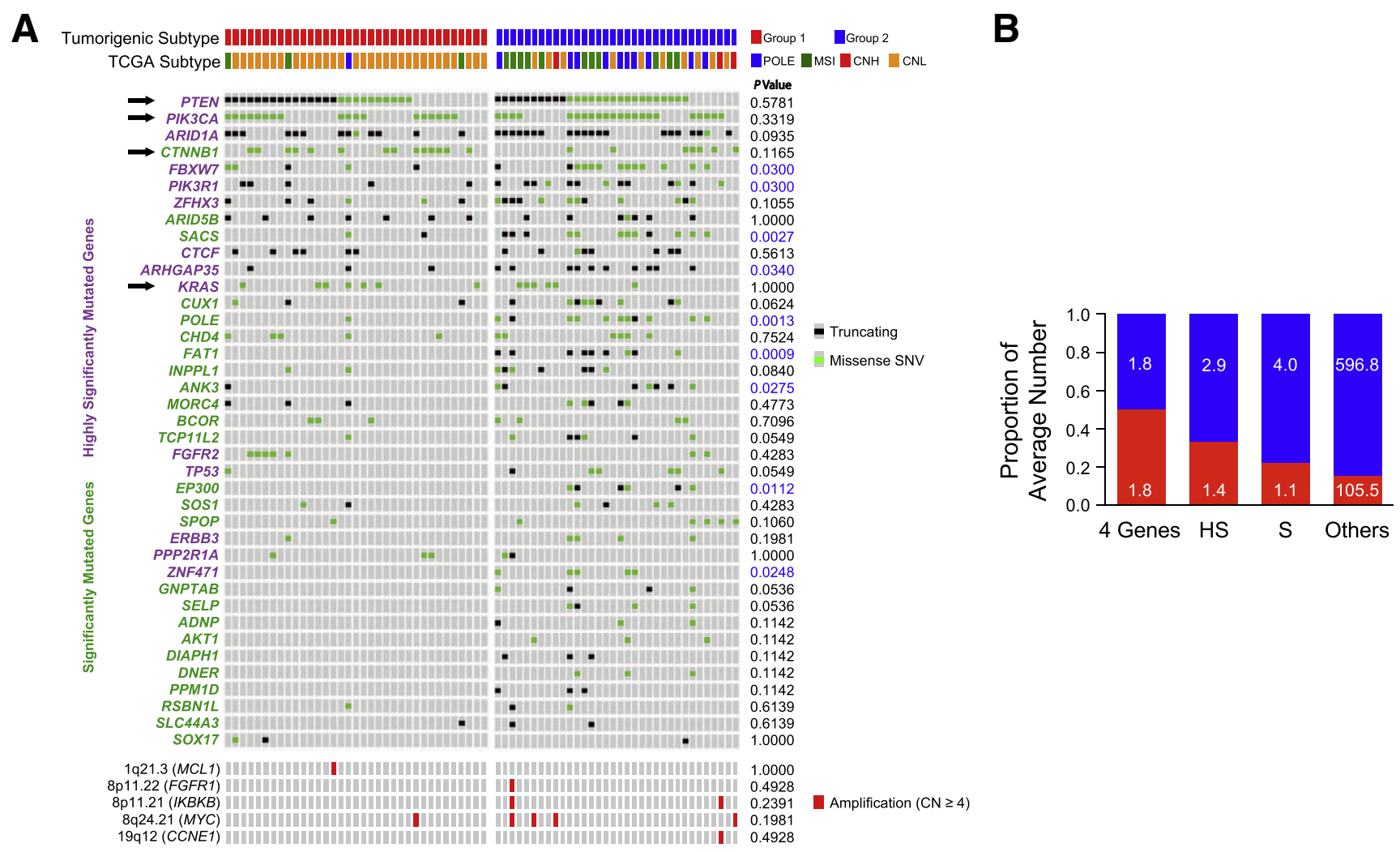

Figure 3 Distribution of driver mutations in group 1 and group 2 carcinomas. A: Oncoprint of driver mutations. Sample labeling for tumorigenic (groups 1 and 2) and The Cancer Genome Atlas (TCGA) subtypes [polymerase $\varepsilon$ mutated (POLE), microsatellite instability (MSI), ${ }^{13}$ copy number high (CNH), and copy number low (CNL)] is shown above the Oncoprints. Top panel: Oncoprint of driver single-nucleotide variants (SNVs)/insertions/deletions (indels) in 35 group 1 and 34 group 2 carcinomas. Highly significantly (HS) and significantly (S) mutated genes follow the assignment in the TumorPortal (http://www.tumorportal. org, last accessed August 27, 2019). Four endometrioid genes (PTEN, PIK3CA, CTNNB1, and KRAS) ${ }^{13,61}$ are indicated by arrows. The detected variants are shown if they were registered at least once in TumorPortal or COSMIC database, had $>0.2$ in the mutant allele frequency after tumor content adjustment, and were detected in more than two samples in the cohort. The $P$ values in binary comparisons with Fisher exact tests are shown on the left. Blue font indicates a significant $P$ value with group 2 enrichment. Bottom panel: Oncoprint of significant copy number alterations detected by GISTIC. The analysis detected only the regions with amplification [copy number $(C N) \geq 4$ ] and not those with homozygous deletion $(C N=0)$. Copy number gain $(C N=3)$ or loss $(C N=1)$ was not regarded as functionally significant in this analysis. The $P$ values in binary comparisons with Fisher exact tests are shown on the left. B: Average number of driver genes (SNVs and indels) per case. Proportions for group 1 and 2 carcinomas are shown in bar plots. The four endometrioid genes (PTEN, PIK3CA, CTNNB1, and KRAS) were not used for calculations of HS or Sgenes in TumorPortal (http://www.tumorportal.org, last accessed August 27, 2019) for SNVs and indels.

\section{Epigenetic Landscape in Group 1 and 2 Carcinomas}

DNA methylation microarray assays were conducted with group 1 and 2 carcinoma samples to classify epigenetic subtypes (Figure 4A). Unsupervised hierarchical clustering of variably methylated $\mathrm{CpG}$ island probes (6963 probes; top $5 \%$ variance) led to the identification of three major clusters (designated ES1, ES2, and ES3) with differential intensities of CpG island methylation (Figure 4A). Most $(9 / 11 ; 81.8 \%)$ of the ES2 cluster comprised group 2 carcinomas, whereas approximately half $(33 / 58 ; 56.9 \%)$ of the ES1 and ES3 clusters comprised group 1 carcinomas (Fisher exact test; $P=0.0234$ ). Because $\mathrm{CpG}$ islands of tumors in the ES2 cluster were the most highly and widely methylated among the three clusters (data not shown), tumors in the ES2 cluster were considered to have a $\mathrm{CpG}$ island methylator phenotype. $^{13}$ Furthermore, five of the eight EEAs displaying $M L H 1$ promoter hypermethylation ( $\beta$ value $>0.5$ ) coincided in the ES2 subtype. Correlating these epigenetic subtypes with TCGA molecular subtypes, significant enrichment of MSI tumors was found among the ES2 cluster (Fisher exact test; $P<0.0001$ ).

To assess characteristic DNA methylation profiles for each tumorigenic subtype, a binary comparison was performed using SAM to compare group 1 and 2 carcinomas. Using 18,034 variably methylated probes at $\mathrm{CpG}$ sites on the array (variance $>0.025$ ), regardless of genomic context (such as promoter, gene body, and untranslated region), all $\mathrm{CpG}$ sites correlated with group 2 carcinomas but not with group 1 carcinomas (SAM $q<0.05$ ) (Figure 4B). Combinatorial correlative analysis with the transcriptome data identified $U B B, C H F R$, $M T E R F$, and $M L H 1$ as epigenetic silencing target genes with group 2 enrichment, implicating an important role for these genes in the tumorigenic program of group 2 EEAs (Figure 4C). 
A

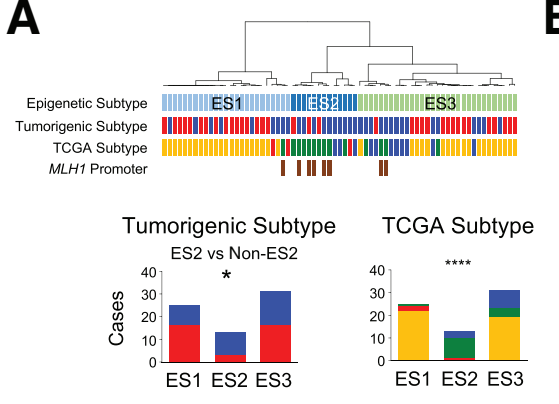

B

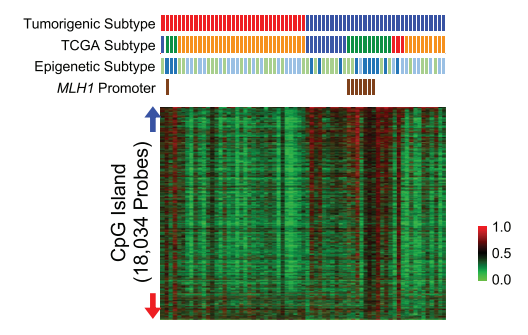

C

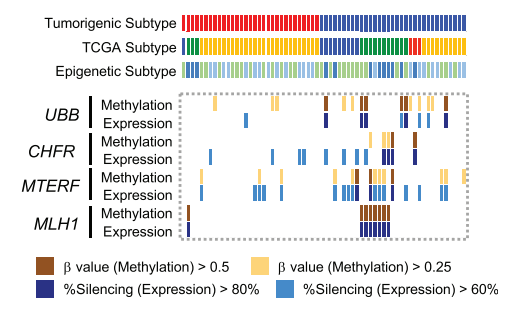

Epigenetic Subtype $\square$ ES1 $\square$ ES2 $\square$ ES3 Tumorigenic Subtype $\square$ Group 1 Group 2
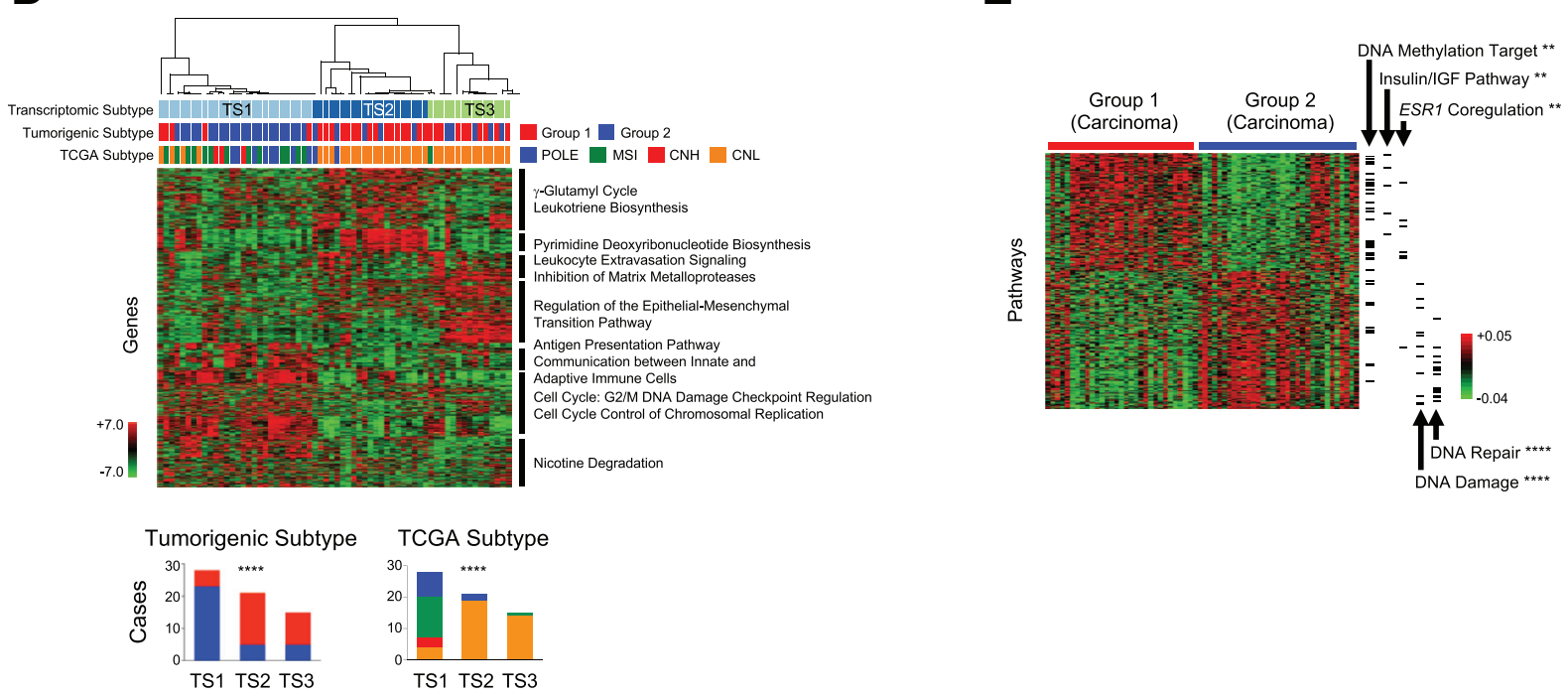

Figure 4 Epigenetic and transcriptomic features of group 1 and 2 endometrial endometrioid adenocarcinomas (EEAs). A: Epigenetic intrinsic subtypes. Epigenetic subtypes were identified through unsupervised hierarchical clustering with variably methylated $6963 \mathrm{CpG}$ island probes (top 5\% variable DNA methylation). Top panel: Sample labeling for epigenetic [epigenetic subtype 1 (ES1), ES2, and ES3], tumorigenic (groups 1 and 2), and The Cancer Genome Atlas (TCGA) subtypes [polymerase $\varepsilon$ mutated (POLE), microsatellite instability (MSI), copy number high (CNH), and copy number low (CNL)] and MLH1 promoter hypermethylation status are shown below the dendrogram. The relationship between tumorigenic (bottom left panel) and TCGA molecular subtype (bottom right panel) with epigenetic subtype in EEA is also shown. A bar represents the number of cases with group 1 and 2 carcinomas or tumors with TCGA molecular subtype assignment in an epigenetic subtype. B: Distinctions in the methylome of group 1 and group 2 carcinoma samples. The result from binary comparisons between group 1 and 2 carcinomas by significance analysis of microarray (SAM) is shown as a heat map of $\beta$ values of probes for variably methylated $\mathrm{CpG}$ islands (18,034 probes with variance $>0.025)$. $\beta$ Values are shown after sorting along SAM scores. Sample labeling for tumorigenic, TCGA, and epigenetic subtypes and MLH1 promoter hypermethylation status are shown above the heat map. Red and blue arrows adjacent to the heat map indicate directions for group 1 and 2 correlations, respectively. C: Extent of DNA methylation and gene silencing for UBB, CHFR, MTERF, and MLH1. Sample labeling for tumorigenic, TCGA, and epigenetic subtypes is also shown above the color map. D: Transcriptomic intrinsic subtypes. Top panel: Heat map for genes used for transcriptomic subtyping is shown. Consensus clustering identifies three transcriptional subgroups [transcriptomic subtype 1 (TS1), TS2, and TS3] in EEAs. The expression levels of genes are shown (green indicates low expression; and red, high expression) with representative gene ontology annotated from Top Canonical Pathways with statistical significance $(P<0.05)$ by Ingenuity Pathway Analysis. Relationship between transcriptomic subtypes with tumorigenic (bottom left panel) and TCGA (bottom right panel) subtypes. A bar represents the number of cases with group 1 and 2 carcinomas or with tumors with TCGA molecular subtype assignment in a transcriptomic subtype. E: Pathway deregulation in group 1 and 2 carcinomas identified by expression profiling. Group 1 and 2 carcinoma-specific enrichment in transcriptomic pathway activities was revealed by binary comparisons of group 1 and 2 carcinomas. Pathway activities with $q<0.05$, derived from SAM analysis of single-sample Gene Set Enrichment Analysis scores, are shown with the heat map. Green and red indicate low and high activities for each gene set, respectively. A total of 1856 and 2180 pathways are highly enriched in group 1 and 2 carcinomas, respectively. Group 1 carcinomas are characterized by the up-regulation of DNA Methylation Target genes, Insulin/Insulin-Like Growth Factor (IGF) Pathway, and ESR1 Coregulation, whereas DNA Damage and DNA Repair related gene sets are more enriched in group 2 carcinomas, with statistical significance by hypergeometric tests. $n=31$ (E, group 1 carcinomas); $n=33$ ( $\mathbf{E}$, group 2 carcinomas). ${ }^{*} P<0.05,{ }^{* *} P<0.01$, and ${ }^{* * * *} P<0.0001$.

\section{Transcriptomic Characterization of Two Distinct Tumorigenic Subtypes in EEA}

After expression microarrays were obtained with Affymetrix U133 Plus 2.0 chip, $K$-means consensus clustering was performed with highly variably expressed genes (top $19 \%$ variably expressed; gene number $=4889$ ). Three transcriptionally intrinsic subtypes (TS1, TS2, and TS3) were identified in EEAs, with seven gene clusters (Figure 4D). TS1, TS2, and TS3 were characterized by the 
expression of cell cycle, pyrimidine deoxyribonucleotide biosynthesis, and epithelial-mesenchymal transition genes, respectively. Group 1 and 2 carcinomas tended to distribute to TS1 and TS2/3 subtypes, respectively (Fisher; $P<0.0001$ ) (Figure 4D). TCGA subtypes also exhibited a skewed distribution, with TS1 and TS2/3 subtypes predominantly allocated to non-CNL (POLE/MSI/CNH) and CNL subtypes, respectively (Figure 4D).

ssGSEA was conducted, and the scores of group 1 and 2 carcinoma samples were compared with SAM in a binary manner. Through pathway analysis, an up-regulation was found in annotations of ESRl coregulation [eg, van 't Veer Breast Cancer ESR1 up - down (UP - DN) and Doane Breast Cancer ESR1 UP - DN; hypergeometric distribution; $P=0.0026]$ and those of insulin/insulin-like growth factor (IGF) pathways in group 1 (eg, Insulin-Like Growth Factor Receptor Binding and IGF1 Pathway; $P=0.0022$, respectively). In group 2, suppression of multiple gene sets of DNA methylation targets (eg, Wang Methylated in Breast Cancer and Weber Methylated in Colon Cancer; $P=0.0010$ ); activation of DNA damage-related gene sets (DNA Damage Response Signal Transduction, Damaged DNA Binding, and DNA Damage Checkpoint; $P<0.0001$ ), and activation of DNA repair-related gene sets (eg, KEGG Base Excision Repair and DNA Repair; $P<0.0001$ ) was found (Figure 4E).

Among 12,406 Molecular Signature DataBase version 5.0 gene sets used in the SAM, there were 14 gene sets related to ER downstream genes: most of these were derived from breast cancer data, with no gene set curated from endometrial cancer or tissue data in Molecular Signature DataBase. Previous studies have identified seven estrogen-induced genes in human in vivo endometrium: IGF1, IGF1R, PGR, KIAA1324, SFRP1, SFRP4, and ALDH1A2. ${ }^{62-64}$ To detect ER signaling in endometrial tumors with ssGSEA, an Endometrial ER Downstream gene set was generated using these seven genes (Figure 5, B and D). The gene set confirmed a significant elevation in estrogen signaling activity in group 1 EEAs (Figure 5B). Spearman correlation analyses between the ssGSEA score for this gene set and the immunoreactivity scores for ER and PR revealed marginal $(P=0.0514$ and $\rho=0.2465)$ and strong $(P<0.0001$ and $\rho=0.5939)$ correlations, respectively. This observation also supports the utility of PR immunoreactivity score as a surrogate marker of estrogen signaling activity.

\section{Hormonal Status of Tumorigenic Subtypes}

Significant differences were identified for age at diagnosis, body mass index, menopausal status, and histologic grade between the two groups (group 1,n=104; group 2, $n=108$ patients). Patients in group 1 were younger at onset, had higher body mass indexes, and were more often premenopausal, whereas those in group 2 had more highgrade carcinomas (Figure 5A). These tendencies remained after selecting only those samples used for genomic analyses (Figure 5A). Furthermore, these tendencies may point to higher concentrations of serum estradiol in patients with group 1 carcinomas (Figure 5C). ${ }^{1-3}$

Transcriptomic and immunohistochemical examinations confirmed up-regulation of ER and PR signaling in group 1 carcinomas compared with group 2 carcinomas (Figures 4E and $5 \mathrm{~B}$ ), consistent with previous observations. ${ }^{65}$ More important, these higher ER/PR protein levels in group 1 carcinomas were also observed in samples from premenopausal and CNL-subtype patients, for whom tumor growth is considered to be largely promoted by estrogen signaling (Figure 5D). ${ }^{13}$ Endometrial estrogen signaling downstream genes exhibited significantly higher activity in group 1 carcinomas than group 2 carcinomas in premenopausal and postmenopausal comparisons for all subtypes, but the statistical significance was not observed for CNL, maybe because of the small number of samples (Figure 5D). Age at diagnosis and body mass index were not different between group 1 and 2 premenopausal patients with CNL tumors. There was thus a difference in the hormonal status of group 1 and 2 tumors, even in estrogen-promoting tumors in the hormonally active phase.

\section{Genomic/Epigenetic/Hormonal Alterations in Transition of Hyperplasia-Carcinoma Sequence}

To examine whether carcinoma acquires genomic and/or epigenetic aberration(s) in the transition between hyperplasia and carcinoma, the mutational burden of paired hyperplasia and carcinoma samples was first compared. Wilcoxon signed rank tests for the number of SNVs, indels, and copy number variants revealed no difference in the mutational burden between hyperplasia and adjacent carcinoma within the same case (Figure 6A). In TCGA subtypes, hyperplasia and carcinoma pairs showed $97.1 \%$ concordance (33/34 pairs) (Figure 6B), along with the identification of shared mutations between hyperplasia and carcinoma. On average, 219.1 of the SNVs/indels were shared, along with 72.7 and 50.1 hyperplasia- and carcinoma-specific SNVs/indels, respectively. Among the highly significantly mutated genes, 1.5 genes were shared, whereas 0.2 driver mutations per sample existed only in carcinoma (Figure 6C). Further narrowing down our analysis to four endometrioid genes (PTEN, PIK3CA, CTNNB1, and $K R A S$ ) increased the number of shared mutations to 1.8 genes. No specific genes with SNVs/indels were differentially and recurrently detected between the pairs. No shifts from subclonal mutations were observed in hyperplasia to clonal mutations in carcinoma, and there was no distinction in the copy number variants, including loss of heterozygosity in tumor suppressor genes, between hyperplasia and carcinoma samples (Figure 6D). Transcriptome and DNA methylome analyses also revealed commonality between pairs of hyperplasia and carcinoma (Figure 6, E and F). Moreover, no statistical distinction with stringent $q$ values was detected in differentially expressed or methylated genes between the pairs (data not shown). Nevertheless, significant down-regulation of ER and PR protein levels and endometrial ER downstream mRNAs were observed in carcinoma compared with 
A
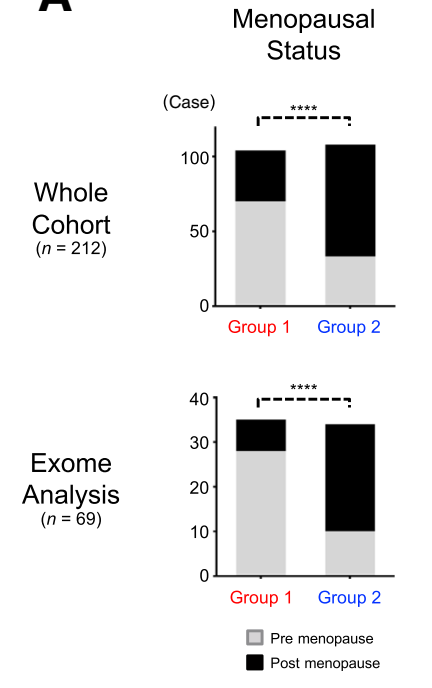

C

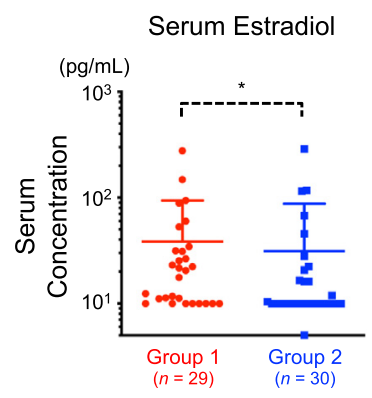

B
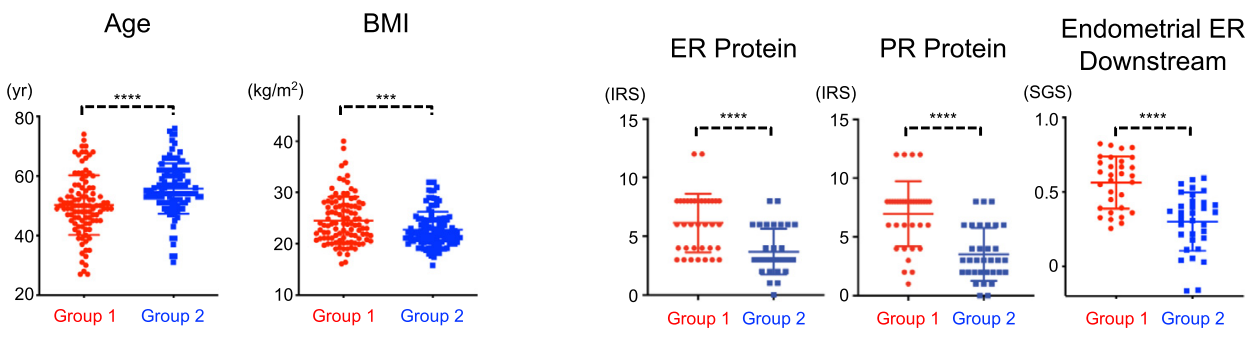
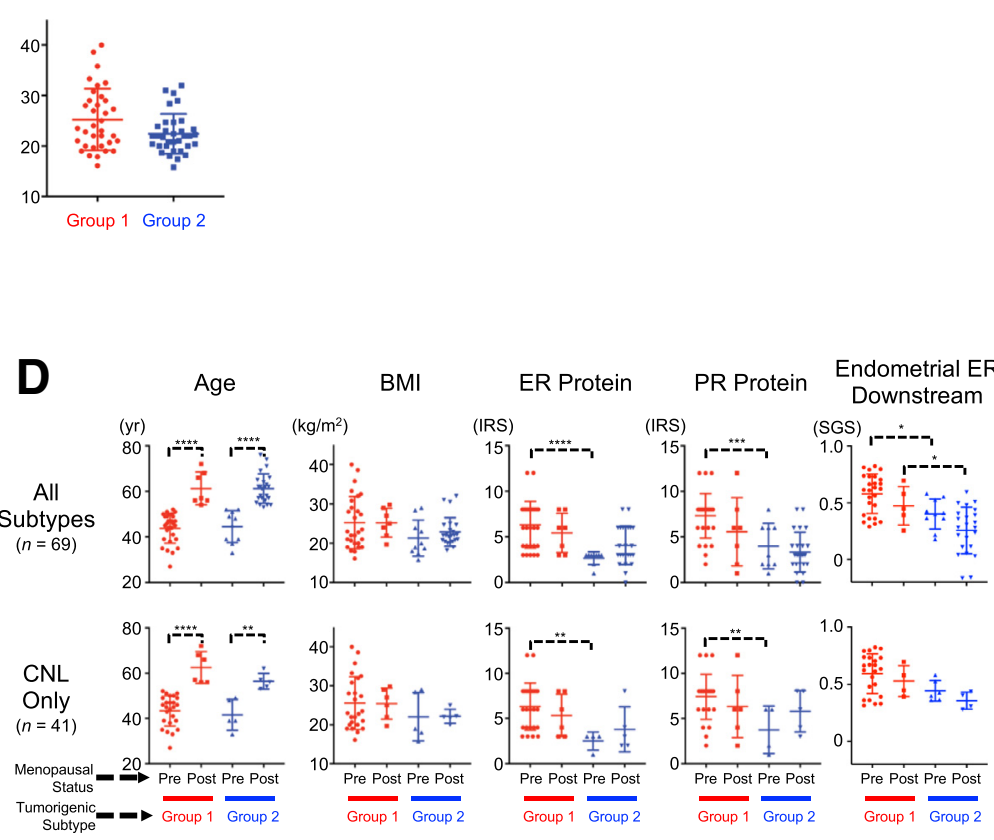

Figure 5 Distinctions in hormonal status between group 1 and 2 carcinoma patients. A: Menopausal status, age at diagnosis, and body mass index (BMI). Top row: Distinctions in the whole cohort with clinicopathologic analysis are shown. Bottom row: Only the results from the samples with genomic analysis are shown. $P$ values were calculated with Fisher exact tests (menopausal status) or with $U$-tests (age and BMI). B: Activity of estrogen signaling. Dot plots represent immunoreactivity scores (IRSs) for estrogen receptor (ER) and progesterone receptor (PR) proteins (left and middle panels) and for single-sample Gene Set Enrichment Analysis score (SGS) of the Endometrial ER Downstream gene set (right panel) in group 1 carcinoma and group 2 carcinoma. Statistical binary comparison was performed with $U$-test. C: Serum estradiol level of group 1 and group 2 patients. Statistical binary comparison was performed with $U$ test. The patients for whom serum estradiol was measured are distinct from the patients whose tumors were rendered to genomic testing and immunostaining of hormonal receptors (Figure 1). D: Age at diagnosis, BMI, IRS of ER and PR, and SGS of the Endometrial ER Downstream gene set in premenopausal or postmenopausal patients between group 1 carcinoma and group 2 carcinoma. The results from tumors of all The Cancer Genome Atlas (TCGA) molecular subtypes and those of only copy number low (CNL) subtype tumors. Statistical binary comparison was performed with $U$-test. $n=212$ in total (104 group 1 and 108 group 2 patients; A, top panels); $n=69$ in total ( 35 group 1 and 34 group 2 patients; $\mathbf{A}$, bottom panels); $n=29$ (C, group 1 patients); $n=30$ (C, group 2 patients); $n=69$ (D, tumors of all TCGA molecular subtypes); $n=41$ (D, tumors of only CNL subtype). ${ }^{*} P<0.05,{ }^{* *} P<0.01,{ }^{* * *} P<0.001$, and $* * * P<0.0001$.

hyperplasia within the paired comparisons (Figure 7, A and B). Overall, these observations suggest that the transition from hyperplasia to carcinoma does not require additional genomic/epigenetic alterations as driver events but is coupled with a decreased dependence on estrogen.

\section{Clonal Evolution in Hyperplasia-Carcinoma Sequence}

Because the pairwise analyses above did not identify when driver mutations are acquired, longitudinal time-course analyses of a series of hyperplasia and carcinoma were conducted from five cases. In a representative case (CU076), the clustering analysis detected four clones with nine clusters during disease development. For this case, a loss of three hyperplasia clones was noted without driver mutations at the time of surgery (time point, 49 months) (Figure 8A) and a dominance of one clone (beige) that had two driver mutations (PTEN and CTNNB1) was noted at time point 0 months (first biopsy sample by endometrial curettage) and acquired additional driver changes over time (SNV in CHD4 and indel in ARID5B) (Figure 8A). Further sequential time-course analyses of the other four cases (Figure 8B) showed the presence of driver mutations in carcinoma clones that were frequently absent in hyperplasia clones. Among the five cases, 10 of the 14 clones-which could not have obtained dominancy at the time of surgery-did not 
A
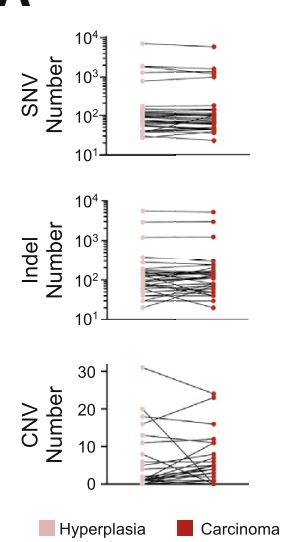

D

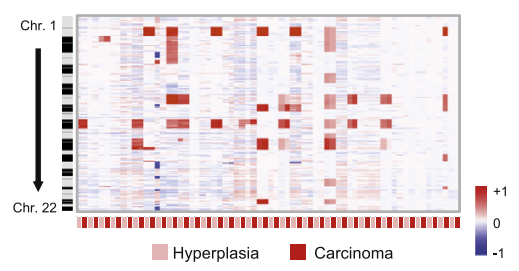

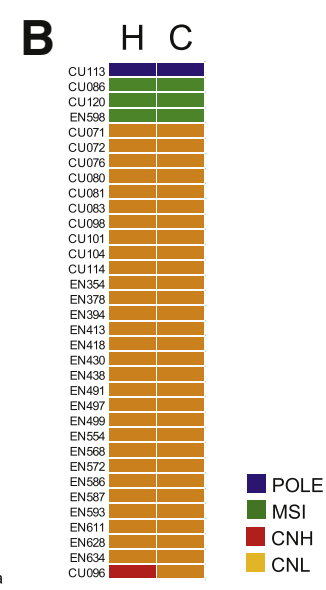

C
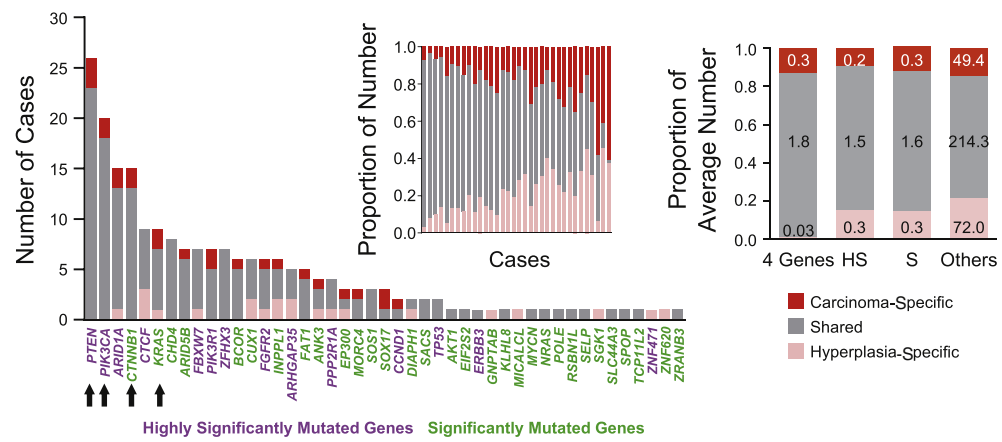

Highly Significantly Mutated Genes Significantly Mutated Genes

E

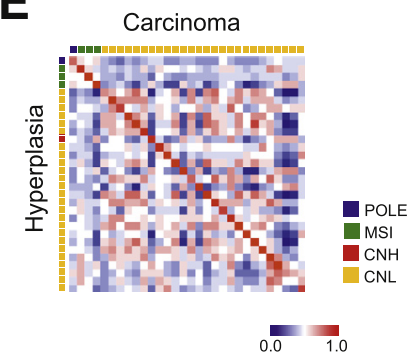

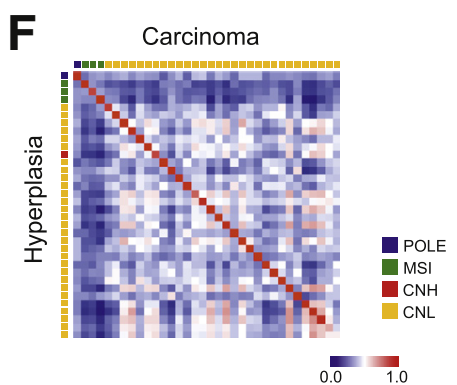

Figure 6 Extensive similarity of genomic, epigenetic, and transcriptomic features in paired hyperplasia (H) and carcinoma (C) samples. A: Mutational burden in hyperplasia and carcinoma. Top panel: Number of single-nucleotide variants (SNVs). Middle panel: Number of insertions/deletions (indels). Bottom panel: Number of copy number variants (CNVs). A statistical binary comparison was performed with Wilcoxon signed rank test. $P$ values are shown beneath the panel. B: The Cancer Genome Atlas (TCGA) molecular subtyping of hyperplasia and carcinoma. TCGA subtypes for hyperplasia and carcinoma are shown with color codes. Case identifiers are shown to the left. Among 34 cases, 33 pairs of hyperplasia and carcinoma showed concordant subtypes; one case (CU096) did not. C: Shared genes between hyperplasia and carcinoma or genes specific to hyperplasia or carcinoma in SNVs and indels. Left panel: Number of cases with SNVs and indels per gene. Genes with SNVs or indels were counted and are shown as a bar plot per sample. Four endometrioid genes (PTEN, PIK3CA, CTNNB1, and $K R A S)^{13,61}$ are indicated by arrows. Middle panel: Proportion of genes for an individual patient. Right panel: Average number of driver genes (SNVs and indels) per case. Proportions for group 1 and 2 carcinomas are shown in bar plots. The four endometrioid genes (PTEN, PIK3CA, CTNNB1, and KRAS) were not used for calculations of highly significantly mutated genes (HS) or significantly mutated genes (S) in TumorPortal (http://www.tumorportal.org, last accessed August 27, 2019) for SNVs and indels. D: Shared segments between hyperplasia and carcinoma or segments specific to hyperplasia or carcinoma in CNVs. A GISTIC output presented as a heat map shows the number of gained (red) or lost (blue) copy number segments. Chromosome (Chr.) position is shown to the left. There was no significantly altered segment in CNVs in pairs of hyperplasia and carcinoma (Wilcoxon signed rank test). E: Similarities in the transcriptome between hyperplasia and carcinoma. Spearman $\rho$ values derived from the correlation analysis are shown as a heat map with labeling of TCGA molecular subtypes. Highly variably expressed (1029) genes (selected by pvclust in R package) were used for the Spearman correlation. F: Similarity in the DNA methylome between hyperplasia and carcinoma. Spearman rho values derived from the correlation analysis are shown in the heat map labeled by TCGA molecular subtype. Highly variably methylated (6642) probes (selected by pvclust in R package) were used for the Spearman correlation. CNH, copy number high; CNL, copy number low; MSI, microsatellite instability; POLE, polymerase $\varepsilon$ mutated.

retain driver alterations. Clones, even with driver mutations, sometimes disappear during the process of clonal evolution. None of the five cases showed an acquisition of copy number alteration, including loss of heterozygosity in tumor suppressor genes, during the sequence (data not shown). These observations imply that driver events are acquired in hyperplasia but not in the transitional phase from hyperplasia to carcinoma.

Time-course immunostaining of PR protein showed a decrease in estrogen signaling activity during the hyperplasiacarcinoma sequence in four of the cases, except EN587, which acquired an ESRI-activating mutation between 41 and 45 months (Figure $7 \mathrm{C}$ and Figure $8 \mathrm{~B}$ ). In three of the four cases, a down-regulation in PR protein occurred during the transition phase from hyperplasia to carcinoma (Figure 7C).

\section{Discussion}

Careful microscopic inspections in previous studies have identified two distinct tumorigenic processes in EEA development, referred to as group 1 and 2 pathways. ${ }^{2,7-12}$ Although this classification cannot be used in practice to assign clinical samples to diagnostic categories, the subtyping scheme provides an understanding of the molecular mechanisms of EEA tumorigenesis. The present research adds several important findings to previous efforts. First, this study shows that the group 1 carcinogenic process is tightly linked with functional alterations, such as the activation of estrogen signaling in cancer cells, probably by extrinsic stimuli. As such, group 1 tumors mostly belong to the CNL subtype. Second, group 2 tumorigenesis is 
A

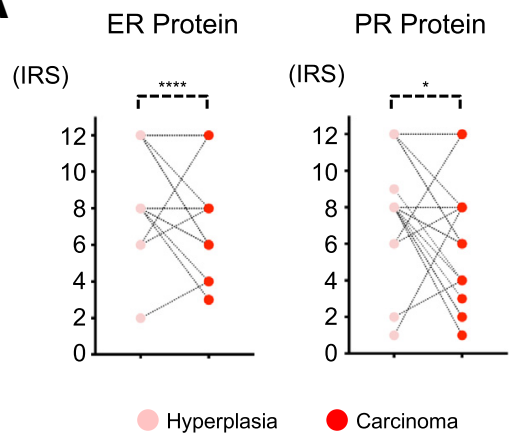

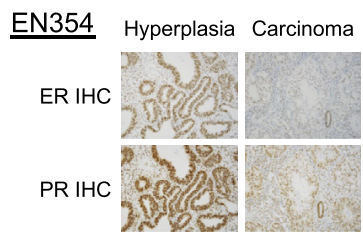

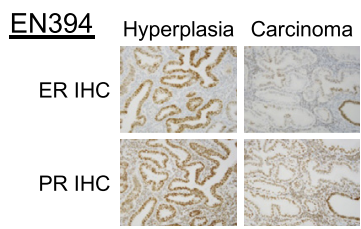

B Endometrial ER

Downstream

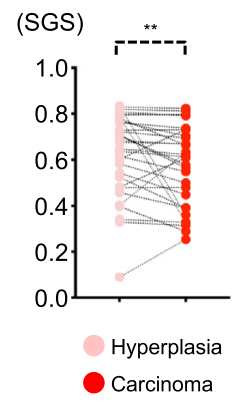

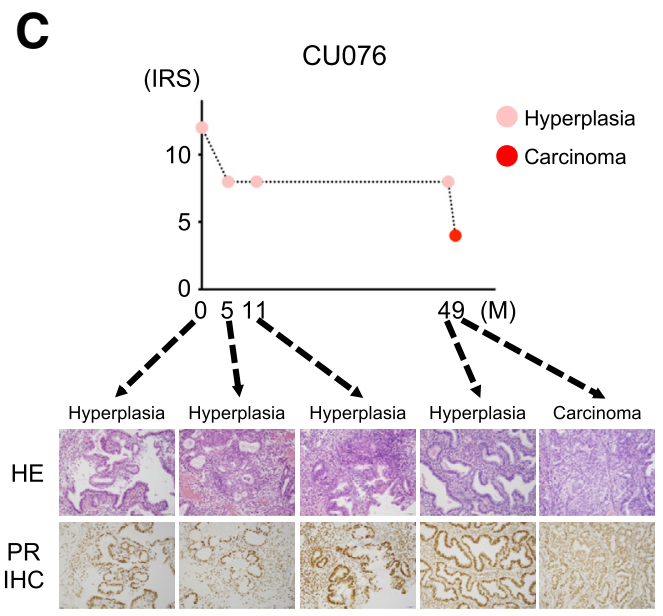
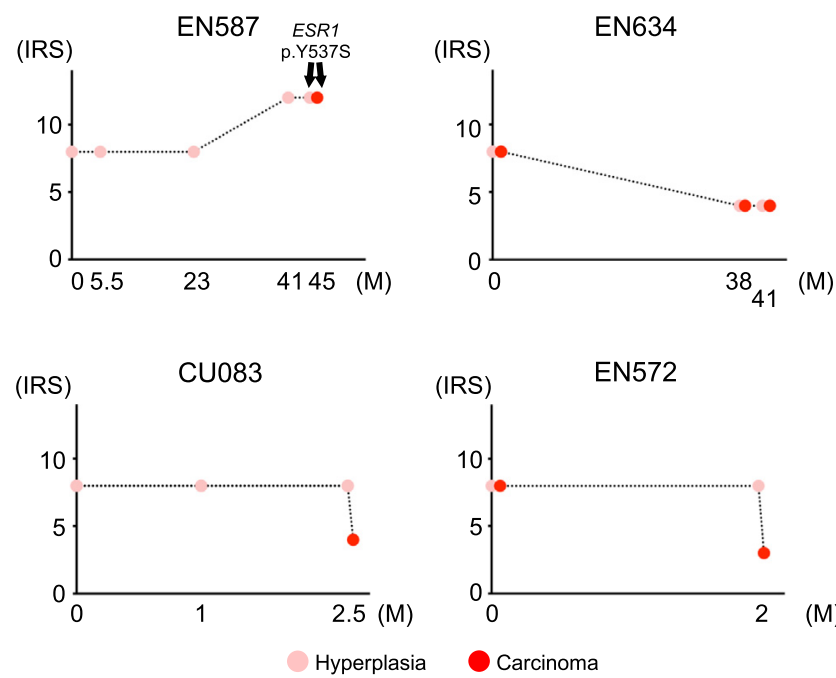

Figure 7 Hormonal status of pairs of hyperplasia and carcinoma and series of tumors in hyperplasia-carcinoma sequence. A: Expression levels of estrogen receptor (ER) and progesterone receptor (PR) proteins in group 1 hyperplasia and carcinoma. The immunoreactivity score (IRS; left panels) and immunohistochemical (IHC) staining (right panels) results are shown for representative cases (EN354 and EN394). Statistical binary comparison was performed with Wilcoxon rank sum test. B: Single-sample Gene Set Enrichment Analysis score (SGS) of the Endometrial ER Downstream gene set in group 1 hyperplasia and carcinoma. C: Time-course presentation of PR IRSs in hyperplasia-carcinoma sequence. Line graphs for PR IRSs for CU076 (top left panel) and EN587, EN634, CU083, and EN572 (right panels) are shown. As a representative case, time-course microscopic images of hematoxylin-eosin staining (HE) and PR IHC staining for CU076 hyperplasia and carcinoma are also shown (bottom left panels). Samples with ESR1-activating mutations are indicated when detected in the line chart. $n=35$ (A and B). ${ }^{*} P<0.05,{ }^{* *} P<0.01$, and ${ }^{* * *} P<0.0001$. Original magnification, $\times 400(\mathbf{A}$ and $\mathbf{C})$. M, months.

associated with a high mutational burden derived from DNA repair deficiency, such as POLE mutations, mismatch repair deficiency, and homologous recombination deficiency, and is frequently accompanied by genome-wide DNA hypermethylation. On the basis of these observations and considering previous studies, the tumorigenic programs of EEA development can be broadly divided into two molecular mechanisms provoked by an excess of unopposed estrogen and by a mutation load comprising multiple forms of DNA repair deficiency (Figure 9).

In the time-course sample analyses, many hyperplasia subclones appeared and disappeared without any identifiable driver mutations, suggesting that the growth of hyperplasia cells is promoted by extrinsic factors, such as estrogen. Driver acquisition seemingly occurs during the evolutionary phase of hyperplasia not at the transitional point from hyperplasia to carcinoma. This hypothesis is not inconsistent with the findings of previous cohort studies using unpaired hyperplasia and carcinoma samples, where there is a lower detectability of several driver mutations in hyperplasia than in carcinoma. ${ }^{19-24}$ Transcriptome and DNA methylome analyses further point to the extensive similarity of each pair of precursor and derivative despite their histologic differentiation. Herein, driver acquisition is assumed as a process of selection and expansion of a hyperplasia subclone. However, there is a discrepancy between the timing of the acquisition of driver genetic changes and that of reduced estrogen dependence (decreased immunoreactivity score of ER/PR and down-regulation of ER downstream gene expression), which occurs in the transition phase from hyperplasia to carcinoma, as previously reported. ${ }^{66,67}$ This observation suggests a molecular mechanism by which reduced estrogen dependence is 
A

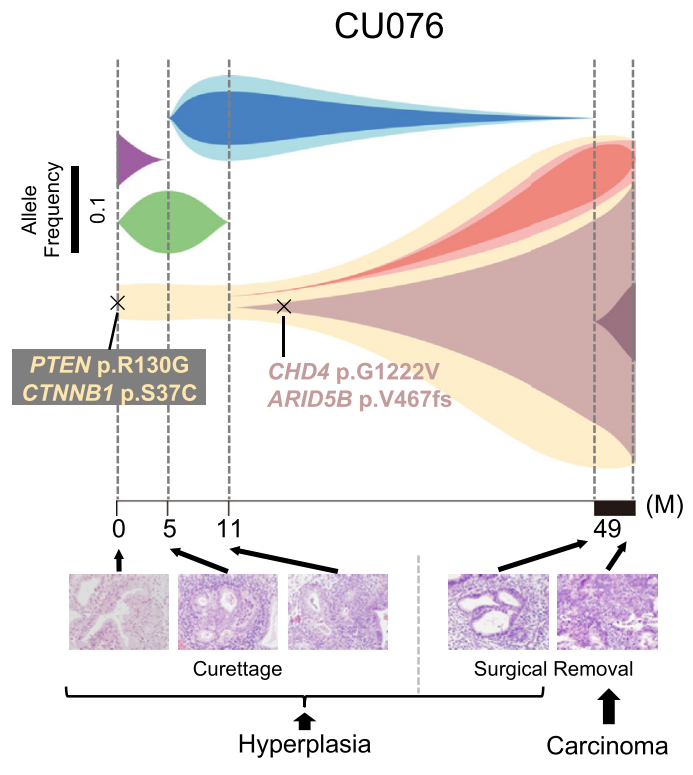

B

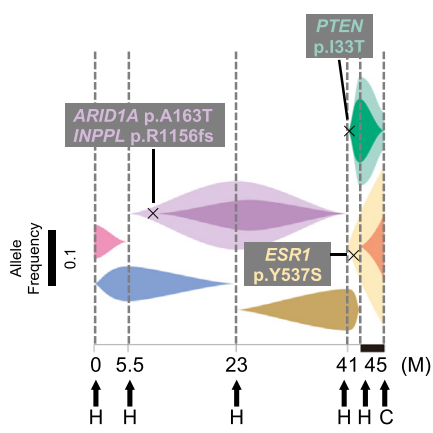

CU083

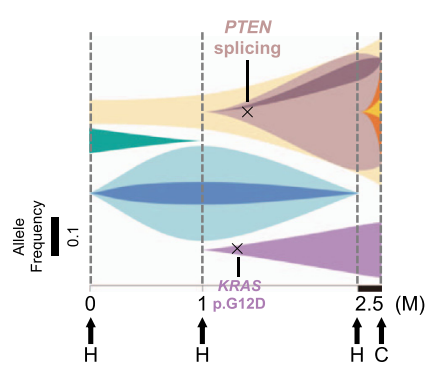

EN634

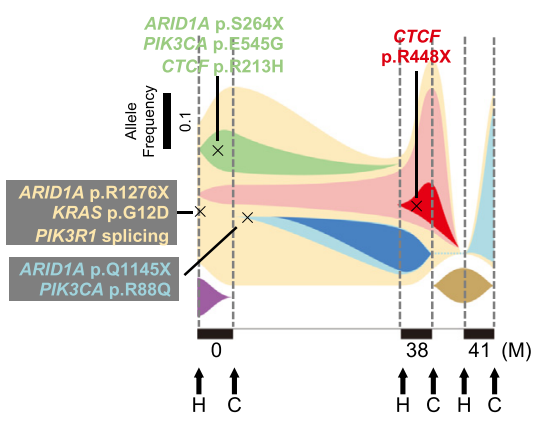

EN572

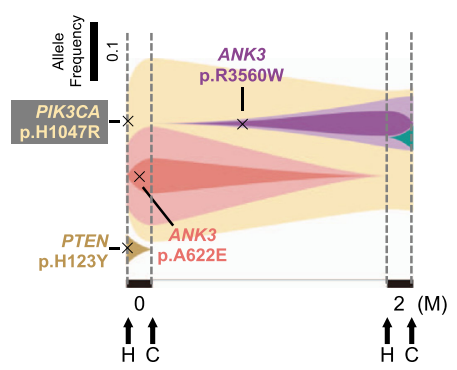

Figure 8 Diagram of clonal architecture in the time course of hyperplasia $(\mathrm{H})$-carcinoma $(\mathrm{C})$ sequence. A: Clonal architecture of hyperplasia and carcinoma in the case, CU076. Clustering analysis detected nine clusters during disease development. A cluster was drawn as a spindle shape or a horn shape, with a color indicating an individual cluster. The dominant clone at the time of surgery is illustrated in beige. The allele frequency for a cluster is reflected as the width at each time point. Time point 0 is defined as the time when the first sample was taken at biopsy by endometrial curettage. The Cancer Genome Atlas (TCGA) driver mutations [highly significantly mutated genes and significantly mutated genes in TumorPortal (http://www.tumorportal.org, last accessed August 27, 2019)] are indicated at the putative time point when a subclone acquired a mutation. Histopathologic findings of tumors are shown beneath the diagram. Dashed lines indicate time points. Hyperplasia and carcinoma samples were taken simultaneously at surgery at time point of 49 months. B: Clonal architecture for EN587, EN634, CU083, and EN572 cases. Nine, eight, nine, and seven clusters (subclones) were detected for the time-course samples for EN587, EN634, CU083, and EN572, respectively. TCGA driver mutations are indicated at the putative time points when a subclone acquired a mutation. The dominant cluster at the time of surgery is shown in beige. A mutation in ESR1 - not included in the list of TCGA driver genes but recently considered as a driver for endometrial cancer ${ }^{13}$ - is shown in EN587. Dashed lines indicate a different sample of either hyperplasia or carcinoma at the same time point. Original magnification, $\times 400$. M, months.

coupled with hyperplasia-carcinoma transformation; however, such a mechanism could not be identified in the current study. More genomic data and/or detailed clinicopathologic information of endometrial hyperplasia would help to understand the mechanism; such information is not currently available in the public databases, including TCGA.

Although the cell of origin has not yet been identified for endometrioid and serous carcinomas, one possible hypothesis is that a common ancestor acquires distinct driver events, such as mutations in PTEN and TP53, which drive tumorigenic programs for endometrioid and serous histologies, respectively. ${ }^{68}$ Although a mutation in PTEN (and/or the other endometrioid drivers) promotes EEA development via endometrial hyperplasia (group 1) or directly from normal atrophic endometria (group 2), as presented in the current study, endometrial serous carcinoma typically develops from normal atrophic endometria and occasionally via endometrial intraepithelial carcinoma as a precursor lesion. ${ }^{69}$

In the tumorigenesis of group 2 endometrioid carcinoma, cancer cells arise in the background of atrophic normal endometrium typically in postmenopausal women. The atrophic endometrium is generally considered to have a low proliferative capacity; however, in a previous study, Ki-67, a standard cell-proliferation marker, was expressed in the atrophic endometria of more than half of the postmenopausal women tested. $^{70}$ Therefore, group 2 carcinoma cells can be derived from atrophic endometrial cells with proliferative potential; albeit, the de novo carcinogenic process thus far remains unclear. Herein, 24 of 34 (70.6\%) group 2 tumors had a high mutational load due to DNA repair deficiency. This high mutational load can provide cells with a chance to acquire driver mutations during tumorigenesis. That the remaining carcinomas possess the CNL phenotype, which is thought to depend on estrogen stimulation, ${ }^{13}$ indicates that a CNL tumor can occur directly from atrophic endometrium but not necessarily through estrogen-dependent hyperplasia. Smaller tumor samples $(<15 \mathrm{~mm}$ along the major axis) were selected, and rigorous and careful microscopic examinations were performed to identify hyperplasia tissue within the entire endometrium to minimize misclassifications between groups 1 and $2 .^{12}$ However, carcinoma can physically take over regions of adjacent hyperplastic tissue, and not identifying these regions 


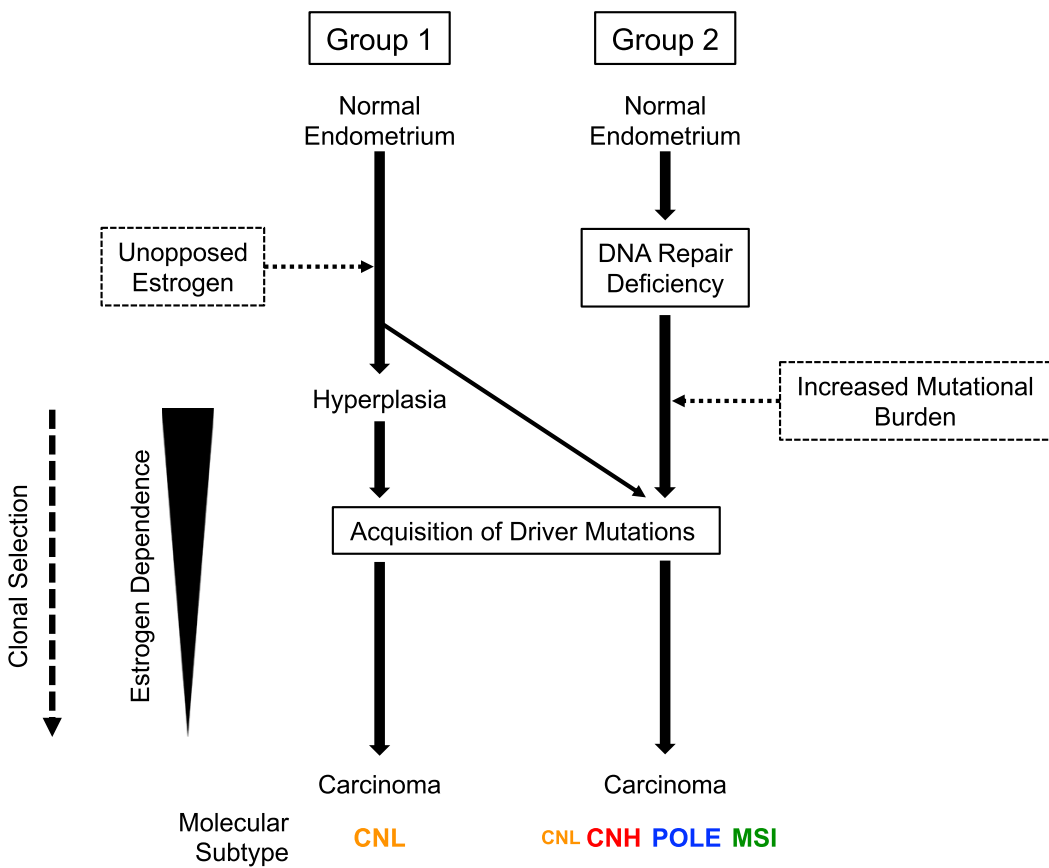

Figure 9 Schematic presentation of two distinct tumorigenic pathways in endometrial endometrioid adenocarcinoma development. CNH, copy number high; CNL, copy number low; MSI, microsatellite instability; POLE, polymerase $\varepsilon$ mutated. of hyperplasia might lead to a misclassification of group 1 CNL carcinomas to group 2. Nevertheless, both postmenopausal and premenopausal group $2 \mathrm{CNL}$ carcinomas had lower expression levels of endometrial estrogen downstream genes accompanied by a down-regulation in ER/PR proteins and, thus, were considered to be less dependent on the estrogen signal. This finding implies that a weak estrogen stimulation, one that is incapable of promoting the hyperplasia-carcinoma sequence, could still promote de novo carcinogenesis from atrophic endometria. Indeed, the serum estradiol concentrations of group 2 patients were lower than those of group 1 patients, supporting this hypothesis.

A previous study ${ }^{71}$ identified two distinct transcriptomic subgroups within the CNL subtype, which may be related to the tumorigenic subtypes in the current study. Among cluster I and cluster II, cluster II is characterized by Wnt pathway activation and enriched CTNNBI-activating mutations. ${ }^{71}$ In the present consensus clustering analysis, TS2 and TS3 were both predominantly of the CNL subtype. TS3 had an up-regulated expression of epithelial-mesenchymal transition related genes and CTNNB1-activating mutations; $63.2 \%$ (12 of 19) of CTNNB1-activating mutations distributed to TS3, which is consistent with the findings that epithelial-mesenchymal transition is triggered by $\beta$-catenin activation in several cancer types. ${ }^{71,72}$ Herein, we consider our TS2 and TS3 are reminiscent of cluster I and cluster II, respectively, in the previous analysis. ${ }^{71}$ In the present consensus clustering analysis, among 31 group 1 carcinomas, 16 samples (51.6\%) and 10 samples (32.3\%) were clustered to TS2 and TS3, respectively, whereas many of the carcinomas in group $2(69.7 \% ; 23 / 33)$ were clustered as TS1. Given that there is no significant difference between group 1 and 2 carcinomas in CTNNB1 mutation positivity, as presented, and in ssGSEA scores of epithelialmesenchymal transition-related gene sets (data not shown), it can be concluded that group 1 and 2 tumorigenic subtypes are mostly independent of the transcriptomic subtype characterized by the CTNNB1-activating mutation and epithelial-mesenchymal transition phenotype.

Only three samples were detected with MSI among the 35 cases of group 1 hyperplasia/carcinomas (8.6\%) (Figure 2D). Many previous studies with limited sample size, typically $<10$ samples, have detected MSI or MLH1 hypermethylation in hyperplasia with variable frequencies $(17 \%$ to $50 \%),{ }^{73-78}$ which may oppose our notion. However, the frequencies were $7.4 \%$ (MSI) and 3.4\% (mismatch repair protein deficiency) in two studies that analyzed $>50$ hyperplasia samples, ${ }^{79,80}$ comparable with the findings of this study. Another study ${ }^{81}$ also supported this finding, showing evidence that carcinomas with MSI arose more frequently in atrophic endometrium.

In breast cancer, molecular subtype is critically determined by the set of driver events, the cell type of origin, and the hormonal environment. ${ }^{82-85}$ In the present study, we unraveled the relations between tumorigenic subtype and molecular subtype, driver changes, and hormonal influences. The cell of origin for EEA in normal endometrium has yet to be elucidated, and its identification will help to greatly enhance our understanding of the detailed molecular mechanisms of these two distinct tumorigenic programs.

\section{Acknowledgments}

We thank Kazuma Kiyotani, Yusuke Nakamura, Kosei Hasegawa, Noriomi Matsumura, Tsukasa Baba, Yasuo 
Uemura, Yoshio Miki, Mitsuaki Yoshida, Yu Imamura, Kazuyoshi Kato, Hidetaka Nomura, Teiichi Motoyama, Nobuhiro Takeshima, and Futoshi Akiyama for helpful discussions; Yukie Nakashima, Megumi Nakai, Rika Nishiko, Junko Kanayama, Akihisa Takahara, Sayuri Amino, Rie Furuya, Yuki Ota, Noriko Yaguchi, Kumiko Sakurai, Miyuki Kogure, Motoyoshi Iwakoshi, and Tomoyo Kakita for technical assistance; Minako Hoshida and Kana Hayashi for administrative assistance; and Rebecca Jackson for editing a draft of the manuscript.

\section{Author Contributions}

Y.S., O.G., and S.M. analyzed the data and wrote the article; Y.T. confirmed the histopathologic diagnoses; N.F. and N.T. analyzed the data; Y.S. and K.H. collected the specimens and provided clinical information; T.N. and S.M. conceived the study and wrote the article.

\section{Supplemental Data}

Supplemental material for this article can be found at https://doi.org/10.1016/j.ajpath.2019.09.022.

\section{References}

1. Bokhman JV: Two pathogenetic types of endometrial carcinoma. Gynecol Oncol 1983, 15:10-17

2. Deligdisch L, Cohen CJ: Histologic correlates and virulence implications of endometrial carcinoma associated with adenomatous hyperplasia. Cancer 1985, 56:1452-1455

3. Amant F, Moerman P, Neven P, Timmerman D, Van Limbergen E, Vergote I: Endometrial cancer. Lancet 2005, 366:491-505

4. Setiawan VW, Yang HP, Pike MC, McCann SE, Yu H, Xiang YB, et al: Type I and II endometrial cancers: have they different risk factors? J Clin Oncol 2013, 31:2607-2618

5. Murali R, Soslow RA, Weigelt B: Classification of endometrial carcinoma: more than two types. Lancet Oncol 2014, 15:e268-e278

6. Suarez AA, Felix AS, Cohn DE: Bokhman redux: endometrial cancer "types" in the 21st century. Gynecol Oncol 2017, 144:243-249

7. Kaku T, Tsukamoto N, Hachisuga T, Tsuruchi N, Sakai K, Hirakawa T, Amada S, Saito T, Kamura T, Nakano H: Endometrial carcinoma associated with hyperplasia. Gynecol Oncol 1996, 60: $22-25$

8. Sivridis E, Fox H, Buckley CH: Endometrial carcinoma: two or three entities? Int J Gynecol Cancer 1998, 8:183-188

9. Ohkawara S, Jobo T, Sato R, Kuramoto H: Comparison of endometrial carcinoma coexisting with and without endometrial hyperplasia. Eur J Gynaecol Oncol 2000, 21:573-577

10. Koul A, Willen R, Bendahl PO, Nilbert M, Borg A: Distinct sets of gene alterations in endometrial carcinoma implicate alternate modes of tumorigenesis. Cancer 2002, 94:2369-2379

11. Geels YP, Pijnenborg JM, van den Berg-van Erp SH, Bulten J, Visscher DW, Dowdy SC, Massuger LF: Endometrioid endometrial carcinoma with atrophic endometrium and poor prognosis. Obstet Gynecol 2012, 120:1124-1131

12. Hasumi K, Sugiyama Y, Sakamoto K, Akiyama F: Small endometrial carcinoma $10 \mathrm{~mm}$ or less in diameter: clinicopathologic and histogenetic study of 131 cases for early detection and treatment. Cancer Med 2013, 2:872-880
13. Cancer Genome Atlas Research Network, Kandoth C, Schultz N, Cherniack AD, Akbani R, Liu Y, Shen H, Robertson AG, Pashtan I, Shen R, Benz CC, Yau C, Laird PW, Ding L, Zhang W, Mills GB, Kucherlapati R, Mardis ER, Levine DA: Integrated genomic characterization of endometrial carcinoma. Nature 2013, 497:67-73

14. Gibson WJ, Hoivik EA, Halle MK, Taylor-Weiner A, Cherniack AD, Berg A, Holst F, Zack TI, Werner HM, Staby KM, Rosenberg M, Stefansson IM, Kusonmano K, Chevalier A, Mauland KK, Trovik J, Krakstad C, Giannakis M, Hodis E, Woie K, Bjorge L, Vintermyr OK, Wala JA, Lawrence MS, Getz G, Carter SL, Beroukhim R, Salvesen HB: The genomic landscape and evolution of endometrial carcinoma progression and abdominopelvic metastasis. Nat Genet 2016, 48:848-855

15. Salvesen HB, Carter SL, Mannelqvist M, Dutt A, Getz G, Stefansson IM, Raeder MB, Sos ML, Engelsen IB, Trovik J, Wik E, Greulich H, Bo TH, Jonassen I, Thomas RK, Zander T, Garraway LA, Oyan AM, Sellers WR, Kalland KH, Meyerson M, Akslen LA, Beroukhim R: Integrated genomic profiling of endometrial carcinoma associates aggressive tumors with indicators of PI3 kinase activation. Proc Natl Acad Sci U S A 2009, 106:4834-4839

16. Dutt A, Salvesen HB, Chen TH, Ramos AH, Onofrio RC, Hatton C, Nicoletti R, Winckler W, Grewal R, Hanna M, Wyhs N, Ziaugra L, Richter DJ, Trovik J, Engelsen IB, Stefansson IM, Fennell T, Cibulskis K, Zody MC, Akslen LA, Gabriel S, Wong KK, Sellers WR, Meyerson M, Greulich H: Drug-sensitive FGFR2 mutations in endometrial carcinoma. Proc Natl Acad Sci U S A 2008, 105:8713-8717

17. Berg A, Hoivik EA, Mjos S, Holst F, Werner HM, Tangen IL, Taylor-Weiner A, Gibson WJ, Kusonmano K, Wik E, Trovik J, Halle MK, Oyan AM, Kalland KH, Cherniack AD, Beroukhim R, Stefansson I, Mills GB, Krakstad C, Salvesen HB: Molecular profiling of endometrial carcinoma precursor, primary and metastatic lesions suggests different targets for treatment in obese compared to non-obese patients. Oncotarget 2015, 6:1327-1339

18. Garcia-Dios DA, Lambrechts D, Coenegrachts L, Vandenput I, Capoen A, Webb PM, Ferguson K; ANECS, Akslen LA, Claes B, Vergote I, Moerman P, Van Robays J, Marcickiewicz J, Salvesen HB, Spurdle AB, Amant F: High-throughput interrogation of PIK3CA, PTEN, KRAS, FBXW7 and TP53 mutations in primary endometrial carcinoma. Gynecol Oncol 2013, 128:327-334

19. Maxwell GL, Risinger JI, Gumbs C, Shaw H, Bentley RC, Barrett JC, Berchuck A, Futreal PA: Mutation of the PTEN tumor suppressor gene in endometrial hyperplasias. Cancer Res 1998, 58: $2500-2503$

20. Hayes MP, Wang H, Espinal-Witter R, Douglas W, Solomon GJ, Baker SJ, Ellenson LH: PIK3CA and PTEN mutations in uterine endometrioid carcinoma and complex atypical hyperplasia. Clin Cancer Res 2006, 12:5932-5935

21. Mutter GL: Altered PTEN expression as a diagnostic marker for the earliest endometrial precancers. J Natl Cancer Inst 2000, 92:924-930

22. Konopka B, Janiec-Jankowska A, Kwiatkowska E, Najmola U, Bidzinski M, Olszewski W, Goluda C: PIK3CA mutations and amplification in endometrioid endometrial carcinomas: relation to other genetic defects and clinicopathologic status of the tumors. Hum Pathol 2011, 42:1710-1719

23. Enomoto T, Fujita M, Inoue M, Rice JM, Nakajima R, Tanizawa O, Nomura T: Alterations of the p53 tumor suppressor gene and its association with activation of the c-K-ras-2 protooncogene in premalignant and malignant lesions of the human uterine endometrium. Cancer Res 1993, 53:1883-1888

24. Sasaki H, Nishii H, Takahashi H, Tada A, Furusato M, Terashima Y, Siegal GP, Parker SL, Kohler MF, Berchuck A, Boyd J: Mutation of the Ki-ras protooncogene in human endometrial hyperplasia and carcinoma. Cancer Res 1993, 53:1906-1910

25. Zauber P, Denehy TR, Taylor RR, Ongcapin EH, Marotta S, SabbathSolitare M: Strong correlation between molecular changes in endometrial carcinomas and concomitant hyperplasia. Int J Gynecol Cancer 2015, 25:863-868 
26. Matias-Guiu X, Catasus L, Bussaglia E, Lagarda H, Garcia A, Pons C, Munoz J, Arguelles R, Machin P, Prat J: Molecular pathology of endometrial hyperplasia and carcinoma. Hum Pathol 2001, 32: $569-577$

27. Russo M, Broach J, Sheldon K, Houser KR, Liu DJ, Kesterson J, Phaeton R, Hossler C, Hempel N, Baker M, Newell JM, Zaino R, Warrick JI: Clonal evolution in paired endometrial intraepithelial neoplasia/atypical hyperplasia and endometrioid adenocarcinoma. Hum Pathol 2017, 67:69-77

28. Stelloo E, Bosse T, Nout RA, MacKay HJ, Church DN, Nijman HW, Leary A, Edmondson RJ, Powell ME, Crosbie EJ, Kitchener HC, Mileshkin L, Pollock PM, Smit VT, Creutzberg CL: Refining prognosis and identifying targetable pathways for highrisk endometrial cancer: a TransPORTEC initiative. Mod Pathol 2015, 28:836-844

29. Talhouk A, McConechy MK, Leung S, Li-Chang HH, Kwon JS, Melnyk N, Yang W, Senz J, Boyd N, Karnezis AN, Huntsman DG, Gilks CB, McAlpine JN: A clinically applicable molecular-based classification for endometrial cancers. $\mathrm{Br} \mathrm{J}$ Cancer 2015, 113: 299-310

30. Hansen JM, Baggerly KA, Wang Y, Wu S, Previs RA, Zand B, Dalton HJ, Hu W, Coleman RL, Sood AK: Homologous recombination deficiency in endometrioid uterine cancer: an unrecognized phenomenon. Gynecol Oncol 2015, 137:21

31. Lee YC, Milne RL, Lheureux S, Friedlander M, McLachlan SA, Martin KL, Bernardini MQ, Smith C, Picken S, Nesci S, Hopper JL, Phillips KA: Risk of uterine cancer for BRCA1 and BRCA2 mutation carriers. Eur J Cancer 2017, 84:114-120

32. Shu CA, Pike MC, Jotwani AR, Friebel TM, Soslow RA, Levine DA, Nathanson KL, Konner JA, Arnold AG, Bogomolniy F, Dao F, Olvera N, Bancroft EK, Goldfrank DJ, Stadler ZK, Robson ME, Brown CL, Leitao MM Jr, Abu-Rustum NR, Aghajanian CA, Blum JL, Neuhausen SL, Garber JE, Daly MB, Isaacs C, Eeles RA, Ganz PA, Barakat RR, Offit K, Domchek SM, Rebbeck TR, Kauff ND: Uterine cancer after risk-reducing salpingo-oophorectomy without hysterectomy in women with BRCA mutations. JAMA Oncol 2016, 2:1434-1440

33. Casey MJ, Bewtra C, Lynch HT, Snyder CL, Stacey M: Endometrial cancers in mutation carriers from hereditary breast ovarian cancer syndrome kindreds: report from the Creighton University Hereditary Cancer Registry with review of the implications. Int J Gynecol Cancer 2015, 25:650-656

34. Jeggo PA, Pearl LH, Carr AM: DNA repair, genome stability and cancer: a historical perspective. Nat Rev Cancer 2016, 16 : $35-42$

35. Tavassoli FA, Devilee P: Pathology and Genetics of Tumours of the Breast and Female Genital Organs. Lyon, France: IARC Press, 2003

36. Creasman W: Revised FIGO staging for carcinoma of the endometrium. Int J Gynaecol Obstet 2009, 105:109

37. Kurman RJ: Blaustein's Pathology of the Female Genital Tract. London: Springer, 2002

38. Silverberg SG, Kurman RJ: Tumors of the Uterine Corpus and Gestational Trophoblastic Disease. Washington, DC: Armed Forces Institute of Pathology, 1992

39. Remmele W, Schicketanz KH: Immunohistochemical determination of estrogen and progesterone receptor content in human breast cancer: computer-assisted image analysis (QIC score) vs. subjective grading (IRS). Pathol Res Pract 1993, 189:862-866

40. Li H, Durbin R: Fast and accurate short read alignment with BurrowsWheeler transform. Bioinformatics 2009, 25:1754-1760

41. DePristo MA, Banks E, Poplin R, Garimella KV, Maguire JR, Hartl C, Philippakis AA, del Angel G, Rivas MA, Hanna M, McKenna A, Fennell TJ, Kernytsky AM, Sivachenko AY, Cibulskis K, Gabriel SB, Altshuler D, Daly MJ: A framework for variation discovery and genotyping using next-generation DNA sequencing data. Nat Genet 2011, 43:491-498

42. Koboldt DC, Zhang Q, Larson DE, Shen D, McLellan MD, Lin L, Miller CA, Mardis ER, Ding L, Wilson RK: VarScan 2: somatic mutation and copy number alteration discovery in cancer by exome sequencing. Genome Res 2012, 22:568-576

43. Cibulskis K, Lawrence MS, Carter SL, Sivachenko A, Jaffe D, Sougnez C, Gabriel S, Meyerson M, Lander ES, Getz G: Sensitive detection of somatic point mutations in impure and heterogeneous cancer samples. Nat Biotechnol 2013, 31:213-219

44. Kakiuchi M, Nishizawa T, Ueda H, Gotoh K, Tanaka A, Hayashi A, Yamamoto S, Tatsuno $\mathrm{K}$, Katoh $\mathrm{H}$, Watanabe $\mathrm{Y}$, Ichimura $\mathrm{T}$, Ushiku T, Funahashi S, Tateishi K, Wada I, Shimizu N, Nomura S, Koike K, Seto Y, Fukayama M, Aburatani H, Ishikawa S: Recurrent gain-of-function mutations of RHOA in diffuse-type gastric carcinoma. Nat Genet 2014, 46:583-587

45. McKenna A, Hanna M, Banks E, Sivachenko A, Cibulskis K, Kernytsky A, Garimella K, Altshuler D, Gabriel S, Daly M, DePristo MA: The Genome Analysis Toolkit: a MapReduce framework for analyzing next-generation DNA sequencing data. Genome Res 2010, 20:1297-1303

46. Magi A, Tattini L, Cifola I, D'Aurizio R, Benelli M, Mangano E, Battaglia C, Bonora E, Kurg A, Seri M, Magini P, Giusti B, Romeo G, Pippucci T, De Bellis G, Abbate R, Gensini GF: EXCAVATOR: detecting copy number variants from whole-exome sequencing data. Genome Biol 2013, 14:R120

47. Mermel CH, Schumacher SE, Hill B, Meyerson ML, Beroukhim R, Getz G: GISTIC2.0 facilitates sensitive and confident localization of the targets of focal somatic copy-number alteration in human cancers. Genome Biol 2011, 12:R41

48. Sathirapongsasuti JF, Lee H, Horst BA, Brunner G, Cochran AJ, Binder S, Quackenbush J, Nelson SF: Exome sequencing-based copy-number variation and loss of heterozygosity detection: ExomeCNV. Bioinformatics 2011, 27:2648-2654

49. Rizvi NA, Hellmann MD, Snyder A, Kvistborg P, Makarov V, Havel JJ, Lee W, Yuan J, Wong P, Ho TS, Miller ML, Rekhtman N, Moreira AL, Ibrahim F, Bruggeman C, Gasmi B, Zappasodi R, Maeda Y, Sander C, Garon EB, Merghoub T, Wolchok JD, Schumacher TN, Chan TA: Cancer immunology: mutational landscape determines sensitivity to PD-1 blockade in non-small cell lung cancer. Science 2015, 348:124-128

50. Boland CR, Thibodeau SN, Hamilton SR, Sidransky D, Eshleman JR, Burt RW, Meltzer SJ, Rodriguez-Bigas MA, Fodde R, Ranzani GN, Srivastava S: A National Cancer Institute Workshop on Microsatellite Instability for cancer detection and familial predisposition: development of international criteria for the determination of microsatellite instability in colorectal cancer. Cancer Res 1998, 58:5248-5257

51. Beroukhim R, Getz G, Nghiemphu L, Barretina J, Hsueh T, Linhart D, Vivanco I, Lee JC, Huang JH, Alexander S, Du J, Kau T, Thomas RK, Shah K, Soto H, Perner S, Prensner J, Debiasi RM, Demichelis F, Hatton C, Rubin MA, Garraway LA, Nelson SF, Liau L, Mischel PS, Cloughesy TF, Meyerson M, Golub TA, Lander ES, Mellinghoff IK, Sellers WR: Assessing the significance of chromosomal aberrations in cancer: methodology and application to glioma. Proc Natl Acad Sci U S A 2007, 104: 20007-20012

52. Lawrence MS, Stojanov P, Mermel CH, Robinson JT, Garraway LA, Golub TR, Meyerson M, Gabriel SB, Lander ES, Getz G: Discovery and saturation analysis of cancer genes across 21 tumour types. Nature 2014, 505:495-501

53. Miller CA, White BS, Dees ND, Griffith M, Welch JS, Griffith OL, Vij R, Tomasson MH, Graubert TA, Walter MJ, Ellis MJ, Schierding W, DiPersio JF, Ley TJ, Mardis ER, Wilson RK, Ding L: SciClone: inferring clonal architecture and tracking the spatial and temporal patterns of tumor evolution. PLoS Comput Biol 2014, 10:e1003665

54. Krzywinski M: Visualizing clonal evolution in cancer. Mol Cell 2016, 62:652-656

55. Subramanian A, Tamayo P, Mootha VK, Mukherjee S, Ebert BL, Gillette MA, Paulovich A, Pomeroy SL, Golub TR, Lander ES, Mesirov JP: Gene set enrichment analysis: a knowledge-based 
approach for interpreting genome-wide expression profiles. Proc Natl Acad Sci U S A 2005, 102:15545-15550

56. Liberzon A, Subramanian A, Pinchback R, Thorvaldsdottir H, Tamayo P, Mesirov JP: Molecular signatures database (MSigDB) 3.0. Bioinformatics 2011, 27:1739-1740

57. Liberzon A, Birger C, Thorvaldsdottir H, Ghandi M, Mesirov JP, Tamayo P: The Molecular Signatures Database (MSigDB) hallmark gene set collection. Cell Syst 2015, 1:417-425

58. Monti S, Savage KJ, Kutok JL, Feuerhake F, Kurtin P, Mihm M, Wu B, Pasqualucci L, Neuberg D, Aguiar RC, Dal Cin P, Ladd C, Pinkus GS, Salles G, Harris NL, Dalla-Favera R, Habermann TM, Aster JC, Golub TR, Shipp MA: Molecular profiling of diffuse large B-cell lymphoma identifies robust subtypes including one characterized by host inflammatory response. Blood 2005, 105: $1851-1861$

59. Wilkerson MD, Hayes DN: ConsensusClusterPlus: a class discovery tool with confidence assessments and item tracking. Bioinformatics 2010, 26:1572-1573

60. Deligdisch L: Morphologic correlates of host response in endometrial carcinoma. Am J Reprod Immunol 1982, 2:54-57

61. Bolivar AM, Luthra R, Mehrotra M, Chen W, Barkoh BA, Hu P, Zhang W, Broaddus RR: Targeted next-generation sequencing of endometrial cancer and matched circulating tumor DNA: identification of plasma-based, tumor-associated mutations in early stage patients. Mod Pathol 2019, 32:405-414

62. Deng L, Shipley GL, Loose-Mitchell DS, Stancel GM, Broaddus R, Pickar JH, Davies PJ: Coordinate regulation of the production and signaling of retinoic acid by estrogen in the human endometrium. J Clin Endocrinol Metab 2003, 88:2157-2163

63. Deng L, Broaddus RR, McCampbell A, Shipley GL, Loose DS, Stancel GM, Pickar JH, Davies PJ: Identification of a novel estrogenregulated gene, EIG121, induced by hormone replacement therapy and differentially expressed in type I and type II endometrial cancer. Clin Cancer Res 2005, 11:8258-8264

64. Westin SN, Broaddus RR, Deng L, McCampbell A, Lu KH, Lacour RA, Milam MR, Urbauer DL, Mueller P, Pickar JH, Loose DS: Molecular clustering of endometrial carcinoma based on estrogeninduced gene expression. Cancer Biol Ther 2009, 8:2126-2135

65. Geels YP, van der Putten LJ, van Tilborg AA, Lurkin I, Zwarthoff EC, Pijnenborg JM, van den Berg-van Erp SH, Snijders MP, Bulten J, Visscher DW, Dowdy SC, Massuger LF: Immunohistochemical and genetic profiles of endometrioid endometrial carcinoma arising from atrophic endometrium. Gynecol Oncol 2015, 137:245-251

66. Bergeron C, Ferenczy A: Oncocytic metaplasia in endometrial hyperplasia and carcinoma. Int J Gynecol Pathol 1988, 7:93-95

67. Hu K, Zhong G, He F: Expression of estrogen receptors ERalpha and ERbeta in endometrial hyperplasia and adenocarcinoma. Int $\mathrm{J}$ Gynecol Cancer 2005, 15:537-541

68. Hubbard SA, Gargett CE: A cancer stem cell origin for human endometrial carcinoma? Reproduction 2010, 140:23-32

69. Crum CP, Nucci MR, Granter SR, Howitt BE, Parast MM, Boyd T, Lee KR, Peters WA: Diagnostic Gynecologic and Obstetric Pathology. Amsterdam: Elsevier Health Sciences, 2017

70. Sivridis E: Proliferative activity in postmenopausal endometrium: the lurking potential for giving rise to an endometrial adenocarcinoma. J Clin Pathol 2004, 57:840-844

71. Liu Y, Patel L, Mills GB, Lu KH, Sood AK, Ding L, Kucherlapati R, Mardis ER, Levine DA, Shmulevich I, Broaddus RR, Zhang W: Clinical significance of CTNNB1 mutation and Wnt pathway activation in endometrioid endometrial carcinoma. J Natl Cancer Inst 2014, 106:dju245
72. Gonzalez DM, Medici D: Signaling mechanisms of the epithelialmesenchymal transition. Sci Signal 2014, 7:re8

73. Catasus L, Machin P, Matias-Guiu X, Prat J: Microsatellite instability in endometrial carcinomas: clinicopathologic correlations in a series of 42 cases. Hum Pathol 1998, 29:1160-1164

74. Levine RL, Cargile CB, Blazes MS, van Rees B, Kurman RJ, Ellenson LH: PTEN mutations and microsatellite instability in complex atypical hyperplasia, a precursor lesion to uterine endometrioid carcinoma. Cancer Res 1998, 58:3254-3258

75. Berends MJ, Hollema H, Wu Y, van Der Sluis T, Mensink RG, ten Hoor KA, Sijmons RH, de Vries EG, Pras E, Mourits MJ, Hofstra RM, Buys CH, Kleibeuker JH, van Der Zee AG: MLH1 and MSH2 protein expression as a pre-screening marker in hereditary and non-hereditary endometrial hyperplasia and cancer. Int $\mathrm{J}$ Cancer 2001, 92:398-403

76. Hardisson D, Moreno-Bueno G, Sanchez L, Sarrio D, Suarez A, Calero F, Palacios J: Tissue microarray immunohistochemical expression analysis of mismatch repair (hMLH1 and hMSH2 genes) in endometrial carcinoma and atypical endometrial hyperplasia: relationship with microsatellite instability. Mod Pathol 2003, 16:1148-1158

77. Kanaya T, Kyo S, Sakaguchi J, Maida Y, Nakamura M, Takakura M, Hashimoto M, Mizumoto Y, Inoue M: Association of mismatch repair deficiency with PTEN frameshift mutations in endometrial cancers and the precursors in a Japanese population. Am J Clin Pathol 2005, 124:89-96

78. Guida M, Sanguedolce F, Bufo P, Di Spiezio Sardo A, Bifulco G, Nappi C, Pannone G: Aberrant DNA hypermethylation of hMLH-1 and $\mathrm{CDKN} 2 \mathrm{~A} / \mathrm{p} 16$ genes in benign, premalignant and malignant endometrial lesions. Eur J Gynaecol Oncol 2009, 30:267-270

79. Esteller M, Catasus L, Matias-Guiu X, Mutter GL, Prat J, Baylin SB, Herman JG: hMLH1 promoter hypermethylation is an early event in human endometrial tumorigenesis. Am J Pathol 1999, 155:1767-1772

80. Lucas E, Chen H, Molberg K, Castrillon DH, Rivera Colon G, Li L, Hinson S, Thibodeaux J, Lea J, Miller DS, Zheng W: Mismatch repair protein expression in endometrioid intraepithelial neoplasia/atypical hyperplasia: should we screen for Lynch syndrome in precancerous lesions? Int J Gynecol Pathol 2018, 38:533-542

81. Honore LH, Hanson J, Andrew SE: Microsatellite instability in endometrioid endometrial carcinoma: correlation with clinically relevant pathologic variables. Int J Gynecol Cancer 2006, 16:1386-1392

82. Ince TA, Richardson AL, Bell GW, Saitoh M, Godar S, Karnoub AE, Iglehart JD, Weinberg RA: Transformation of different human breast epithelial cell types leads to distinct tumor phenotypes. Cancer Cell 2007, 12:160-170

83. Keller PJ, Arendt LM, Skibinski A, Logvinenko T, Klebba I, Dong S, Smith AE, Prat A, Perou CM, Gilmore H, Schnitt S, Naber SP, Garlick JA, Kuperwasser C: Defining the cellular precursors to human breast cancer. Proc Natl Acad Sci U S A 2012, 109: 2772-2777

84. Wang Y, Waters J, Leung ML, Unruh A, Roh W, Shi X, Chen K, Scheet P, Vattathil S, Liang H, Multani A, Zhang H, Zhao R, Michor F, Meric-Bernstam F, Navin NE: Clonal evolution in breast cancer revealed by single nucleus genome sequencing. Nature 2014, 512:155-160

85. Early Breast Cancer Trialists' Collaborative Group, Peto R, Davies C, Godwin J, Gray R, Pan HC, Clarke M, Cutter D, Darby S, McGale P, Taylor C, Wang YC, Bergh J, Di Leo A, Albain K, Swain S, Piccart M, Pritchard K: Comparisons between different polychemotherapy regimens for early breast cancer: meta-analyses of long-term outcome among 100,000 women in 123 randomised trials. Lancet 2012, 379:432-444 